\title{
Homophymamide A, Heterodetic Cyclic Tetrapeptide from a Homophymia sp. Marine Sponge: A Cautionary Note on Configurational Assignment of Peptides That Contain a Ureido Linkage
}

Daichi Kanki, ${ }^{\dagger}$ Shohei Nakamukai,${ }^{\dagger}$ Y usuke Ogura, ${ }^{\ddagger}$ Hirosato Takikawa, ${ }^{\ddagger}$ Yuji Ise,${ }^{\S}$ Yasuhiro Morii, Nobuhiro Yamawaki, ${ }^{\wedge}$ Tomohiro Takatani, ${ }^{\wedge}$ Osamu Arakawa, Shigeru Okada, ${ }^{\dagger}$ and Shigeki Matsunaga*,†

${ }^{\dagger}$ Laboratory of Aquatic Natural Products Chemistry, Graduate School of Agricultural and Life Sciences, The University of Tokyo, 1-1-1 Bunkyo-ku, Tokyo 113-8657, Japan

Department of Applied Biological Cheimstry, Graduate School of Agricultural and Life Sciences, The University of Tokyo, 1-1-1 Bunkyo-ku, Tokyo 113-8657, Japan

${ }^{\S}$ Sesoko Station, University of the Ryukyus, 3442 Sesoko, Motobu Okinawa 905-0227, Japan

'Graduate School of Fisheries Science and Environmental Studies, Nagasaki University, Nagasaki 852-8521, Japan

\section{Table of contents}

Table S1. ${ }^{1} \mathrm{H}$ and ${ }^{13} \mathrm{C}$ NMR data for the major conformer of homophymamide A (1) in DMSO- $d_{6}$ Table S2. ${ }^{1} \mathrm{H}$ and ${ }^{13} \mathrm{C}$ NMR data for the minor conformer of homophymamide A (1) in DMSO- $d_{6}$ Table S3. ${ }^{1} \mathrm{H}$ and ${ }^{13} \mathrm{C}$ NMR data for the synthetic homophymamide A (1) in DMSO- $d_{6}$

Table S4. ${ }^{1} \mathrm{H}$ and ${ }^{13} \mathrm{C}$ NMR data for the synthetic L-Arg(No)-CO-D-Lys(Z) methyl ester (2) in DMSO- $d_{6}$ Figure S1. ${ }^{1} \mathrm{H}$ NMR spectrum $(600 \mathrm{MHz})$ of homophymamide A (1) in DMSO- $d_{6}$ Figure S2. ${ }^{13} \mathrm{C}$ NMR spectrum $(150 \mathrm{MHz})$ of homophymamide A (1) in DMSO- $d_{6}$ Figure S3. COSY spectrum (600 MHz) of homophymamide A (1) in DMSO- $d_{6}$ Figure S4. COSY spectrum (600 MHz) of homophymamide A (1) in DMSO- $d_{6}$ (aliphatic region) Figure S5. HSQC spectrum (600 MHz) of homophymamide A (1) in DMSO- $d_{6}$ Figure S6. TOCSY spectrum $(600 \mathrm{MHz})$ of homophymamide A (1) in DMSO- $d_{6}$ Figure S7. TOCSY spectrum $(600 \mathrm{MHz})$ of homophymamide A (1) in DMSO- $d_{6}$ (amide proton region) Figure S8. HMBC spectrum (600 MHz) of homophymamide A (1) in DMSO- $d_{6}$ Figure S9. HMBC spectrum (600 MHz) of homophymamide A (1) in DMSO- $d_{6}$ (amide carbonyl region) Figure S10. NOESY spectrum $(600 \mathrm{MHz})$ of homophymamide A (1) in DMSO- $d_{6}$ Figure S11. NOESY spectrum $(600 \mathrm{MHz})$ of homophymamide A (1) in DMSO- $d_{6}$ (amide proton region) Figure S12. ${ }^{1} \mathrm{H}$ NMR spectrum $(600 \mathrm{MHz})$ of synthetic homophymamide A (1) in DMSO- $d_{6}$ Figure S13. ${ }^{13} \mathrm{C}$ NMR spectrum $(150 \mathrm{MHz})$ of synthetic homophymamide A (1) in DMSO- $d_{6}$ Figure S14. HSQC spectrum $(600 \mathrm{MHz})$ of synthetic homophymamide A (1) in DMSO- $d_{6}$ Figure S15. ${ }^{1} \mathrm{H}$ NMR spectrum $(500 \mathrm{MHz})$ of a 1:1 mixture of natural product and synthetic homophymamide A (1) in DMSO- $d_{6}$

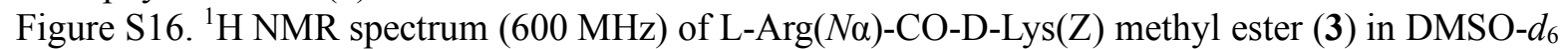
Figure S17. ${ }^{13} \mathrm{C}$ NMR spectrum $(150 \mathrm{MHz})$ of L-Arg $(N \alpha)$-CO-D-Lys(Z) methyl ester (3) in DMSO- $d_{6}$

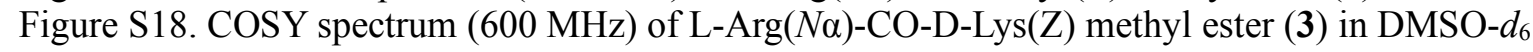
Figure S19-S23. LCMS chromatograms of Marfey's derivatives

Figure S24. Carboxypeptidase B inhibition assay of 1

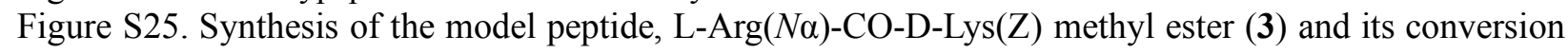
to the hydantoin 4

Figure S26. LCMS chromatogram of the Marfey's derivative of hydantoin 4. 
Table S1. ${ }^{1} \mathrm{H}$ and ${ }^{13} \mathrm{C}$ NMR data for the major conformer of homophymamide A (1) in DMSO- $d_{6}$

\begin{tabular}{|c|c|c|c|c|c|}
\hline position & $\delta_{\mathrm{C}, \text { type }}$ & $\delta_{\mathrm{H}}$ & mult $(J$ in $\mathrm{Hz})$ & NOESY (inter-residual) & $\mathrm{HMBC}^{b}$ \\
\hline \multicolumn{6}{|l|}{$\overline{\text { Arg }}$} \\
\hline 1 & 175.6, C & & & & \\
\hline 2 & $54.1, \mathrm{CH}$ & 3.75 & $\mathrm{~m}$ & Lys, $\alpha-\mathrm{NH}$ & 1 \\
\hline 3 & $30.8, \mathrm{CH}_{2}$ & 1.56 & $\mathrm{~m}$ & & \\
\hline $4 a$ & 25.3, $\mathrm{CH}_{2}$ & 1.45 & $\mathrm{~m}$ & & \\
\hline $4 b$ & & 1.38 & $\mathrm{~m}$ & & \\
\hline 5 & $40.3, \mathrm{CH}_{2}$ & 3.01 & brd (2.2) & & \\
\hline$\alpha-\mathrm{NH}$ & & 6.05 & br s & Lys, $2, \alpha-\mathrm{NH}$; Ala, NH & \\
\hline$\delta-\mathrm{NH}$ & & 9.45 & br s & & \\
\hline guanidino & $157.5^{a}, \mathrm{C}$ & & & & \\
\hline \multicolumn{6}{|l|}{ Ureido } \\
\hline NHCONH & $156.9^{a}, \mathrm{C}$ & & & & \\
\hline \multicolumn{6}{|l|}{ Lys } \\
\hline 1 & $172.4, \mathrm{C}$ & & & & \\
\hline 2 & 53.9, $\mathrm{CH}$ & 3.86 & br s & Arg, $\alpha-\mathrm{NH} ;$ Ala, NH & \\
\hline $3 a$ & $31.3, \mathrm{CH}_{2}$ & 1.51 & $\mathrm{~m}$ & Ala, NH & \\
\hline $3 b$ & & 1.32 & $\mathrm{~m}$ & Arg, $\alpha$-NH; Ala, NH & \\
\hline $4 a$ & 23.3, $\mathrm{CH}_{2}$ & 1.30 & $\mathrm{~m}$ & & \\
\hline $4 b$ & & 1.22 & $\mathrm{~m}$ & & \\
\hline $5 \mathrm{a}$ & 28.7, $\mathrm{CH}_{2}$ & 1.46 & $\mathrm{~m}$ & & \\
\hline $5 b$ & & 1.20 & $\mathrm{~m}$ & & \\
\hline $6 a$ & $38.7, \mathrm{CH}_{2}$ & 3.36 & $\mathrm{~m}$ & Ile, 2 & \\
\hline $6 \mathrm{~b}$ & & 2.83 & br s & Ile, 2 & \\
\hline$\alpha-\mathrm{NH}$ & & 6.38 & br s & Arg, 2, $\alpha-\mathrm{NH}$; Ala: NH & \\
\hline$\varepsilon-\mathrm{NH}$ & & 8.08 & br s & Ile, $2,3,4$ ', NH & \\
\hline \multicolumn{6}{|l|}{ Ala } \\
\hline 1 & $172.2, \mathrm{C}$ & & & & \\
\hline 2 & $44.3, \mathrm{CH}$ & 4.58 & quint (7.0) & NMeLeu, 2, 3, 4, 5', $N$-Me ; Ile, NH & $1,3,1$ (Lys) \\
\hline 3 & $18.3, \mathrm{CH}_{3}$ & 1.09 & $\mathrm{~d}(6.5)$ & NMeLeu, 2 & 1,2 \\
\hline NH & & 7.95 & br s & Arg, $\alpha$-NH; Lys, 2, $\alpha$-NH; Ile, NH; NMeLeu, 2 & \\
\hline \multicolumn{6}{|l|}{$N$-Me-Leu } \\
\hline 1 & $169.5, \mathrm{C}$ & & & & \\
\hline 2 & $58.6, \mathrm{CH}$ & 4.18 & br s & Ala, 2, 3, NH; Ile, NH & 1 \\
\hline $3 a$ & $37.8, \mathrm{CH}_{2}$ & 1.89 & $\mathrm{~m}$ & Ala, 2 & $5^{\prime}$ \\
\hline $3 b$ & & 1.48 & $\mathrm{~m}$ & Ala, 2 & $5,5^{\prime}$ \\
\hline 4 & $25.3, \mathrm{CH}$ & 1.40 & $\mathrm{~m}$ & Ala, 2 & \\
\hline 5 & $23.1, \mathrm{CH}_{3}$ & 0.89 & $\mathrm{~d}(6.6)$ & & $3,4,5^{\prime}$ \\
\hline $5^{\prime}$ & $22.1, \mathrm{CH}_{3}$ & 0.92 & $\mathrm{~d}(6.4)$ & Ala, 2 & $3,4,5$ \\
\hline$N-\mathrm{Me}$ & $30.0, \mathrm{CH}_{3}$ & 2.68 & $\mathrm{~s}$ & Ala, 2; Ile, 4, 4', 5, NH & 2, 1(Ala) \\
\hline \multicolumn{6}{|l|}{ Ile } \\
\hline 1 & $170.4, \mathrm{C}$ & & & & \\
\hline 2 & $57.6, \mathrm{CH}$ & 4.07 & br s & Lys, $6 \mathrm{~b}, \varepsilon-\mathrm{NH}$ & $1,3,3 ', 4,1$ (NMeLeu) \\
\hline 3 & $35.0, \mathrm{CH}$ & 1.89 & $\mathrm{~m}$ & Lys, $\varepsilon-\mathrm{NH}$ & 5 \\
\hline $4 a$ & 24.3, $\mathrm{CH}_{2}$ & 1.42 & $\mathrm{~m}$ & NMeLeu, $N$-Me & 5 \\
\hline $4 b$ & & 1.06 & $\mathrm{~m}$ & NMeLeu, $N$-Me & $3,3 ', 5$ \\
\hline $4^{\prime}$ & $15.4, \mathrm{CH}_{3}$ & 0.77 & $\mathrm{~d}(6.8)$ & Lys, $\varepsilon-\mathrm{NH}$; NMeLeu, $N$-Me & $2,3,4$ \\
\hline 5 & $10.3, \mathrm{CH}_{3}$ & 0.80 & $\mathrm{t}(7.3)$ & NMeLeu, $N$-Me & 3,4 \\
\hline $\mathrm{NH}$ & & 7.73 & br s & Lys, $\varepsilon-\mathrm{NH} ; \mathrm{NMeLeu,} \mathrm{2,} \mathrm{N-Me;} \mathrm{Ala,} \mathrm{2,} \mathrm{NH}$ & 1(NMeLeu) \\
\hline
\end{tabular}

${ }^{a}$ Assignments were made judging from the chemical shift values (references cited in ref 1 in the main text).

${ }^{b}$ Intra residual correlations are shown with carbon number; inter residual correlations are shown in parentheses. 
Table S2. ${ }^{1} \mathrm{H}$ and ${ }^{13} \mathrm{C}$ NMR data for the minor conformer of homophymamide A (1) in DMSO- $d_{6}$

\begin{tabular}{|c|c|c|}
\hline position & $\delta_{\mathrm{C}}{ }^{a}$, type & $\delta_{\mathrm{H}}$ \\
\hline \multicolumn{3}{|l|}{$\overline{\text { Arg }}$} \\
\hline 2 & $54.3, \mathrm{CH}$ & 3.72 \\
\hline 3 & $30.8, \mathrm{CH}_{2}$ & 1.59 \\
\hline $4 a$ & 25.3, $\mathrm{CH}_{2}$ & 1.45 \\
\hline $4 b$ & & 1.38 \\
\hline 5 & $40.3, \mathrm{CH}_{2}$ & 3.01 \\
\hline$\alpha-\mathrm{NH}$ & & 6.18 \\
\hline$\delta$-NH & & 9.45 \\
\hline \multicolumn{3}{|l|}{ Lys } \\
\hline 2 & 53.9, $\mathrm{CH}$ & 3.95 \\
\hline $3 a$ & $31.6, \mathrm{CH}_{2}$ & 1.60 \\
\hline $3 b$ & & 1.47 \\
\hline $4 a$ & $20.9, \mathrm{CH}_{2}$ & 1.16 \\
\hline $4 b$ & & 1.09 \\
\hline $5 \mathrm{a}$ & 29.1, $\mathrm{CH}_{2}$ & 1.28 \\
\hline $6 a$ & 37.5, $\mathrm{CH}_{2}$ & 3.14 \\
\hline $6 b$ & & 3.02 \\
\hline$\alpha-\mathrm{NH}$ & & 6.82 \\
\hline$\varepsilon-\mathrm{NH}$ & & 7.54 \\
\hline \multicolumn{3}{|l|}{ Ala } \\
\hline 2 & $45.5, \mathrm{CH}$ & 4.58 \\
\hline 3 & $17.1, \mathrm{CH}_{3}$ & 1.17 \\
\hline $\mathrm{NH}$ & & 7.40 \\
\hline \multicolumn{3}{|l|}{$N$-Me-Leu } \\
\hline 2 & $66.3, \mathrm{CH}$ & 3.57 \\
\hline $3 a$ & $36.9, \mathrm{CH}_{2}$ & 2.04 \\
\hline $3 b$ & & 1.63 \\
\hline 4 & $24.8, \mathrm{CH}$ & 1.46 \\
\hline 5 & 21.9, $\mathrm{CH}_{3}$ & 0.89 \\
\hline $5^{\prime}$ & 21.9, $\mathrm{CH}_{3}$ & 0.89 \\
\hline$N-\mathrm{Me}$ & 39.7, $\mathrm{CH}_{3}$ & 3.20 \\
\hline \multicolumn{3}{|c|}{ Ile } \\
\hline 2 & 57.4, $\mathrm{CH}$ & 4.04 \\
\hline 3 & $35.9, \mathrm{CH}$ & 1.88 \\
\hline $4 a$ & $24.5, \mathrm{CH}_{2}$ & 1.34 \\
\hline $4 b$ & & 1.01 \\
\hline $4^{\prime}$ & $15.5, \mathrm{CH}_{3}$ & 0.77 \\
\hline 5 & $11.4, \mathrm{CH}_{3}$ & 0.80 \\
\hline $\mathrm{NH}$ & & 7.68 \\
\hline
\end{tabular}

${ }^{a}$ Chemical shifts were determined from the HSQC spectrum. 
Table S3. ${ }^{1} \mathrm{H}$ and ${ }^{13} \mathrm{C}$ NMR data for the synthetic homophymamide A (1) in DMSO- $d_{6}$

\begin{tabular}{|c|c|c|c|c|c|}
\hline \multirow[b]{2}{*}{ position } & \multicolumn{2}{|c|}{ major conformer } & \multirow[b]{2}{*}{ mult $(J$ in $\mathrm{Hz})$} & \multicolumn{2}{|c|}{ minor conformer } \\
\hline & $\delta_{\mathrm{C}}$, type & $\delta_{\mathrm{H}}$ & & $\delta_{\mathrm{C}},{ }^{a}$ type & $\delta_{\mathrm{H}}$ \\
\hline \multicolumn{6}{|l|}{ Arg } \\
\hline 1 & 175.6, C & & & & \\
\hline 2 & $54.1, \mathrm{CH}$ & 3.76 & $\mathrm{q}(5.7)$ & $54.3, \mathrm{CH}$ & 3.73 \\
\hline 3 & $30.7, \mathrm{CH}_{2}$ & 1.56 & $\mathrm{~m}$ & $30.8, \mathrm{CH}_{2}$ & 1.60 \\
\hline $4 a$ & 25.2, $\mathrm{CH}_{2}$ & 1.46 & $\mathrm{~m}$ & 25.2, $\mathrm{CH}_{2}$ & 1.46 \\
\hline $4 b$ & & 1.38 & $\mathrm{~m}$ & & 1.38 \\
\hline 5 & $40.5, \mathrm{CH}_{2}$ & 3.03 & $\mathrm{~m}$ & $40.5, \mathrm{CH}_{2}$ & 3.03 \\
\hline$\alpha-\mathrm{NH}$ & & 6.05 & $\mathrm{~d}(4.8)$ & & 6.17 \\
\hline$\delta-\mathrm{NH}$ & & 9.36 & brs & & 9.36 \\
\hline guanidino & 157.2, C & & & & \\
\hline \multicolumn{6}{|l|}{ Lys } \\
\hline 1 & $172.4, \mathrm{C}$ & & & & \\
\hline 2 & $53.8, \mathrm{CH}$ & 3.86 & brs & $53.8, \mathrm{CH}$ & 3.96 \\
\hline $3 a$ & $31.3, \mathrm{CH}_{2}$ & 1.50 & $\mathrm{~m}$ & $31.5, \mathrm{CH}_{2}$ & 1.61 \\
\hline $3 b$ & & 1.30 & $\mathrm{~m}$ & & 1.45 \\
\hline $4 a$ & 23.3, $\mathrm{CH}_{2}$ & 1.29 & $\mathrm{~m}$ & $20.8, \mathrm{CH}_{2}$ & 1.16 \\
\hline $4 \mathrm{~b}$ & & 1.22 & $\mathrm{~m}$ & & 1.08 \\
\hline $5 \mathrm{a}$ & 28.8, $\mathrm{CH}_{2}$ & 1.46 & $\mathrm{~m}$ & 29.2, $\mathrm{CH}_{2}$ & 1.28 \\
\hline $5 b$ & & 1.20 & $\mathrm{~m}$ & $37.5, \mathrm{CH}_{2}$ & 3.18 \\
\hline $6 a$ & $38.7, \mathrm{CH}_{2}$ & 3.39 & brd (12.3) & & 3.01 \\
\hline $6 b$ & & 2.80 & brq & & \\
\hline$\alpha-\mathrm{NH}$ & & 6.34 & $\mathrm{~d}(3.72)$ & & $\mathrm{ND}^{b}$ \\
\hline$\varepsilon-\mathrm{NH}$ & & 8.07 & brs & & 6.72 \\
\hline \multicolumn{6}{|l|}{ Ureido } \\
\hline NHCONH & $156.8, \mathrm{C}$ & & & & \\
\hline \multicolumn{6}{|l|}{ Ala } \\
\hline 1 & 172.2, C & & & & \\
\hline 2 & $44.4, \mathrm{CH}$ & 4.58 & $q(6.3)$ & $45.4, \mathrm{CH}$ & 4.59 \\
\hline 3 & $18.3, \mathrm{CH}_{3}$ & 1.09 & $\mathrm{~d}(6.2)$ & 17.1, $\mathrm{CH}_{3}$ & 1.16 \\
\hline $\mathrm{NH}$ & & 7.93 & $\mathrm{~d}(6.3)$ & & $\mathrm{ND}^{b}$ \\
\hline \multicolumn{6}{|l|}{$N$-Me-Leu } \\
\hline 1 & $169.5, \mathrm{C}$ & & & & \\
\hline 2 & $58.6, \mathrm{CH}$ & 4.19 & $\mathrm{dd}(4.3,9.2)$ & $66.4, \mathrm{CH}$ & 3.57 \\
\hline $3 a$ & 37.7, $\mathrm{CH}_{2}$ & 1.90 & $\mathrm{~m}$ & $37.0, \mathrm{CH}_{2}$ & 2.05 \\
\hline $3 b$ & & 1.49 & $\mathrm{~m}$ & & 1.64 \\
\hline 4 & $25.2, \mathrm{CH}$ & 1.41 & $\mathrm{~m}$ & $24.8, \mathrm{CH}$ & 1.46 \\
\hline 5 & 23.1, $\mathrm{CH}_{3}$ & 0.90 & $\mathrm{~d}(6.8)$ & 21.9, $\mathrm{CH}_{3}$ & 0.90 \\
\hline $5^{\prime}$ & $22.1, \mathrm{CH}_{3}$ & 0.92 & d (6.4) & 21.9, $\mathrm{CH}_{3}$ & 0.90 \\
\hline$N-\mathrm{Me}$ & 29.9, $\mathrm{CH}_{3}$ & 2.68 & & $39.8, \mathrm{CH}_{3}$ & 3.20 \\
\hline \multicolumn{6}{|l|}{ Ile } \\
\hline 1 & $170.5, \mathrm{C}$ & & & & \\
\hline 2 & $57.5, \mathrm{CH}$ & 4.08 & $\mathrm{t}(8.9)$ & $57.3, \mathrm{CH}$ & 4.04 \\
\hline 3 & $35.0, \mathrm{CH}$ & 1.89 & $\mathrm{~m}$ & $36.0, \mathrm{CH}$ & 1.87 \\
\hline $4 a$ & 24.3, $\mathrm{CH}_{2}$ & 1.41 & $\mathrm{~m}$ & $24.5, \mathrm{CH}_{2}$ & 1.34 \\
\hline $4 b$ & & 1.06 & $\mathrm{~m}$ & & 1.01 \\
\hline $4^{\prime}$ & $15.4, \mathrm{CH}_{3}$ & 0.77 & d (6.6) & $15.4, \mathrm{CH}_{3}$ & 0.78 \\
\hline 5 & $10.3, \mathrm{CH}_{3}$ & 0.80 & $\mathrm{t}(7.3)$ & $11.3, \mathrm{CH}_{3}$ & 0.80 \\
\hline $\mathrm{NH}$ & & 7.68 & $\mathrm{~d}(7.8)$ & & $\mathrm{ND}^{b}$ \\
\hline
\end{tabular}

${ }^{a}$ Chemical shifts were determined from the HSQC spectrum.

${ }^{b}$ Signals were obscured due to broadening. 
Table S4. ${ }^{1} \mathrm{H}$ and ${ }^{13} \mathrm{C}$ NMR data for the synthetic L-Arg(No)-CO-D-Lys(Z) methyl ester (2) in DMSO- $d_{6}$

\begin{tabular}{|c|c|c|c|}
\hline position & $\delta_{\mathrm{C}}$ & $\delta_{\mathrm{H}}$ & mult $(J$ in $\mathrm{Hz})$ \\
\hline \multicolumn{4}{|l|}{ Arg } \\
\hline 1 & 175.5 & & \\
\hline 2 & 53.8 & 3.73 & $\mathrm{q}(6.1)$ \\
\hline 3 & 30.9 & 1.53 & $\mathrm{~m}$ \\
\hline 4 & 25.1 & 1.42 & $\mathrm{~m}$ \\
\hline 5 & 40.4 & 3.04 & br q (6.6) \\
\hline$\alpha-\mathrm{NH}$ & & 6.07 & $\mathrm{~d}(6.5)$ \\
\hline$\delta-\mathrm{NH}$ & & 9.27 & br s \\
\hline $\mathrm{OMe}$ & 51.3 & 3.58 & $\mathrm{~s}$ \\
\hline guanidino & 157.1 & & \\
\hline \multicolumn{4}{|l|}{ ureido } \\
\hline & 156.9 & & \\
\hline \multicolumn{4}{|l|}{ Lys } \\
\hline 1 & 173.9 & & \\
\hline 2 & 52.4 & 4.04 & $\mathrm{dt}(5.9,7.9)$ \\
\hline 3 & 31.4 & 1.52 & $\mathrm{~m}$ \\
\hline 4 & 22.4 & 1.26 & $\mathrm{~m}$ \\
\hline 5 & 28.9 & 1.38 & $\mathrm{~m}$ \\
\hline 6 & 38.8 & 2.96 & $\mathrm{q}(6.8)$ \\
\hline$\alpha-\mathrm{NH}$ & & 6.76 & $\mathrm{~d}(7.9)$ \\
\hline$\varepsilon-\mathrm{NH}$ & & 7.16 & br s \\
\hline \multicolumn{4}{|l|}{ Z } \\
\hline $\mathrm{CO}$ & 156.0 & & \\
\hline $\mathrm{CH}_{2}$ & 65.2 & 5.00 & $\mathrm{~s}$ \\
\hline 1 & 137.2 & & \\
\hline 2 & 127.5 & 7.33 & $\mathrm{~m}$ \\
\hline 3 & 128.2 & 7.35 & $\mathrm{~m}$ \\
\hline 4 & 127.6 & 7.29 & $\mathrm{~m}$ \\
\hline
\end{tabular}




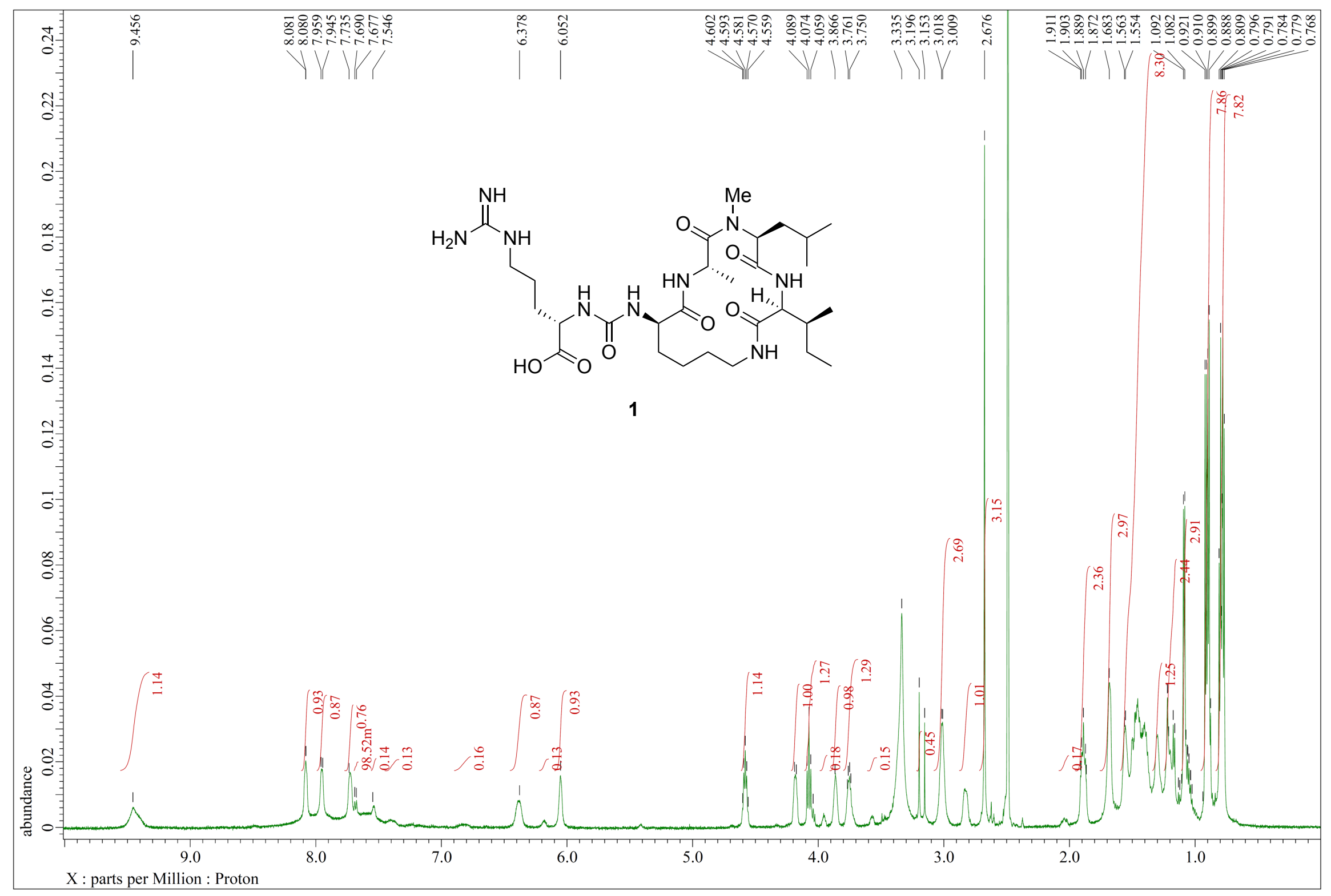

Figure S1. ${ }^{1} \mathrm{H}$ NMR spectrum (600 MHz) of homophymamide A (1) in DMSO- $d_{6}$ 


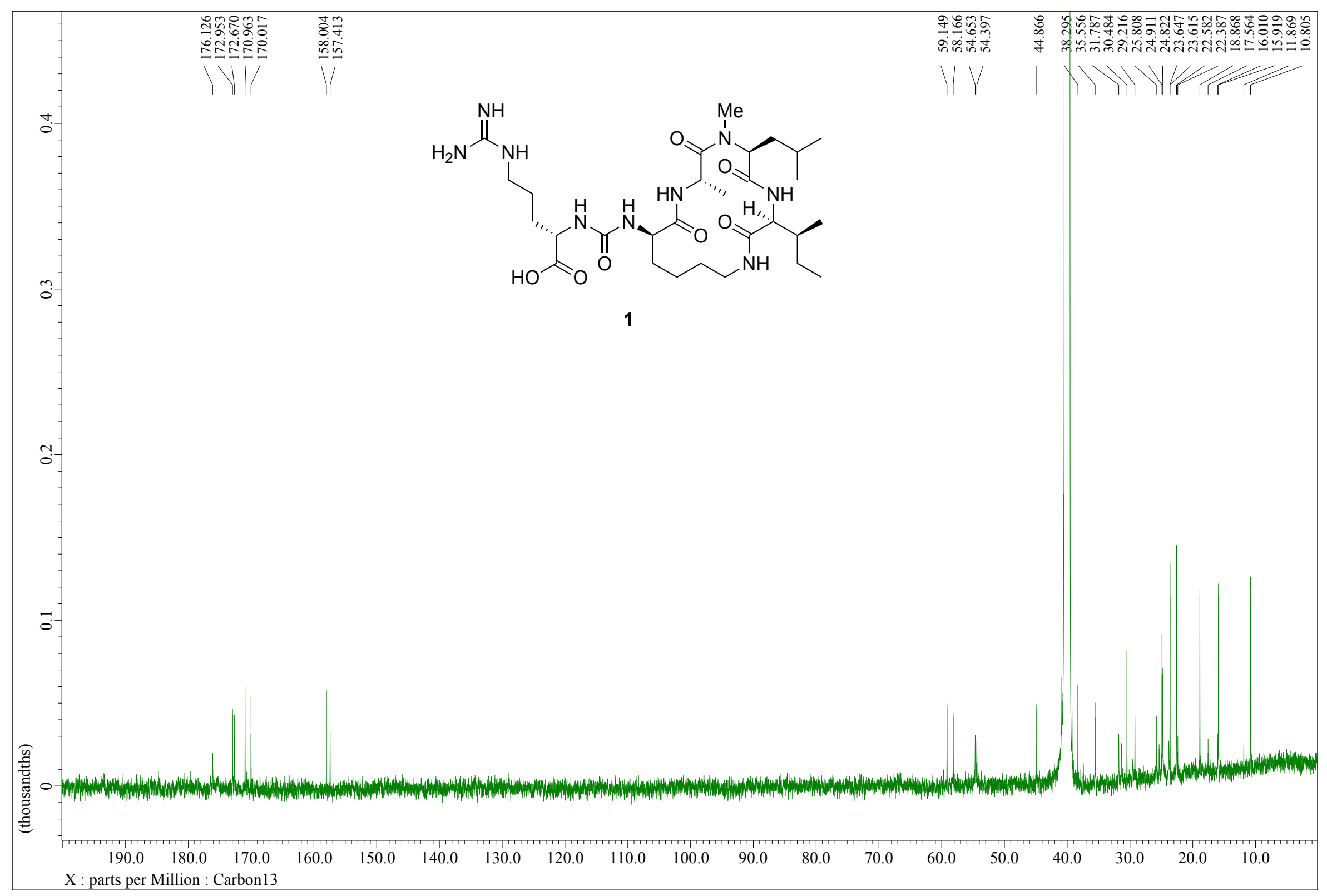

Figure S2. ${ }^{13} \mathrm{C}$ NMR spectrum (150 MHz) of homophymamide A (1) in DMSO- $d_{6}$ 


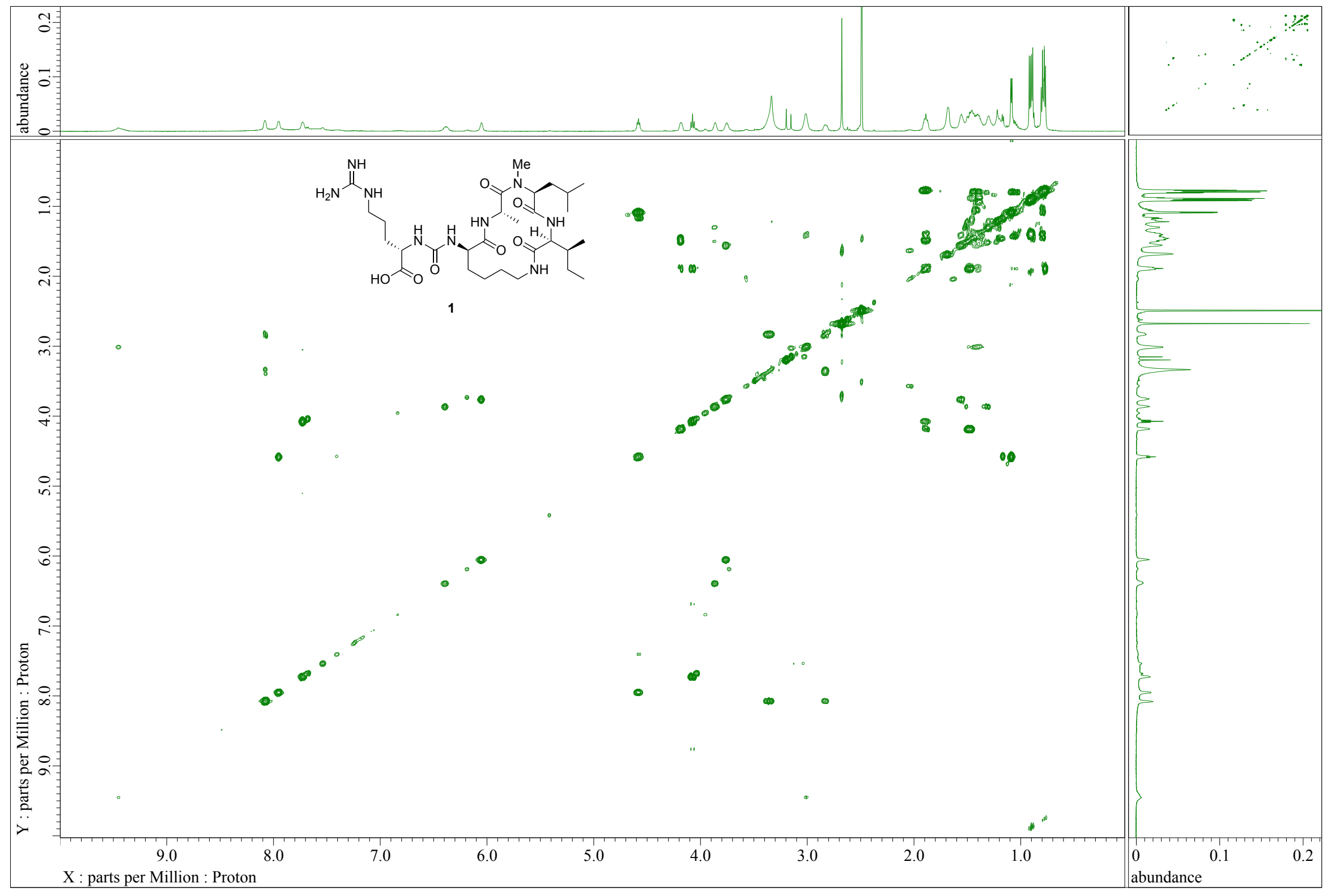

Figure S3. COSY spectrum (600 MHz) of homophymamide A (1) in DMSO- $d_{6}$ 


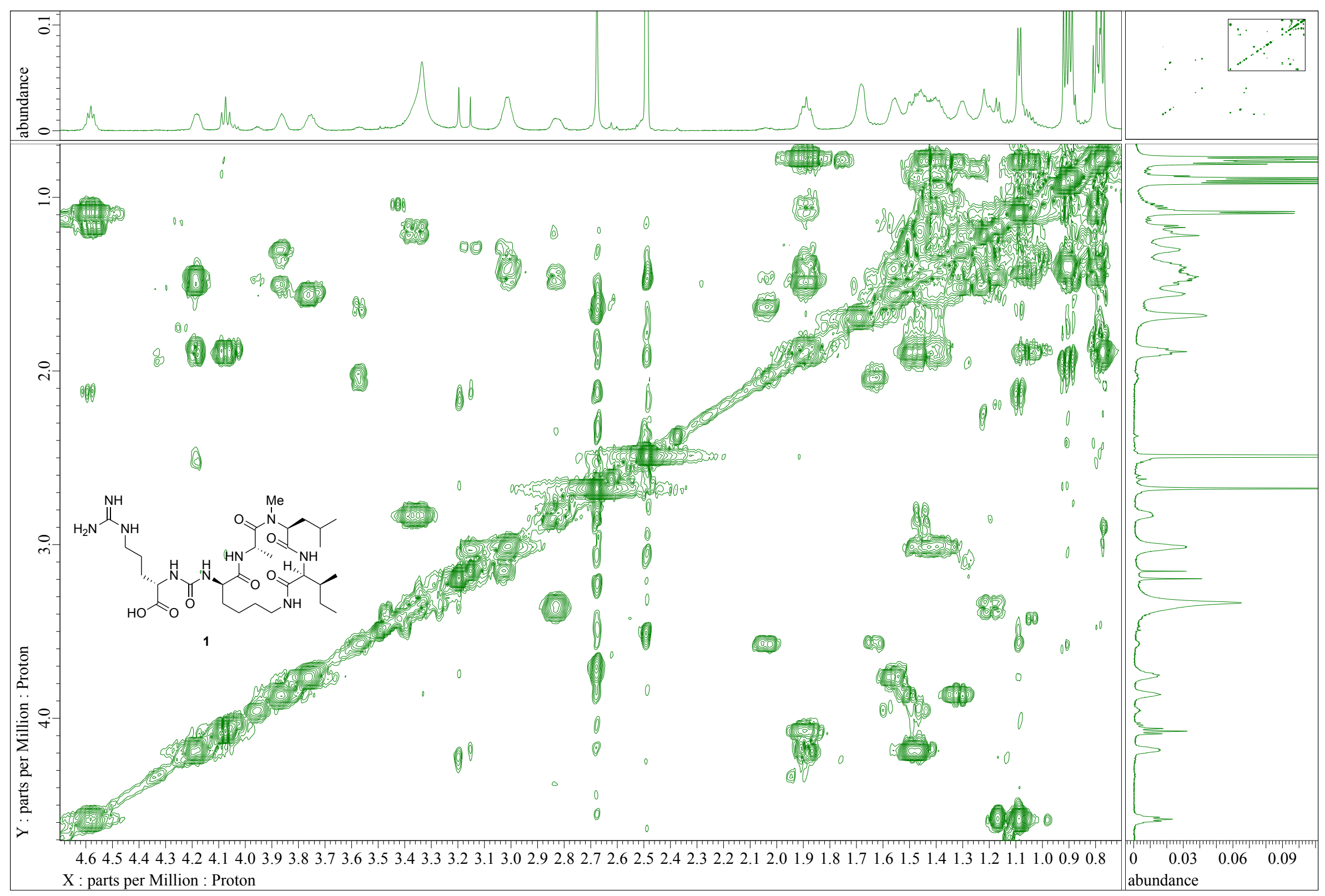

Figure S4. COSY spectrum (600 MHz) of homophymamide A (1) in DMSO- $d_{6}$ (aliphatic region) 


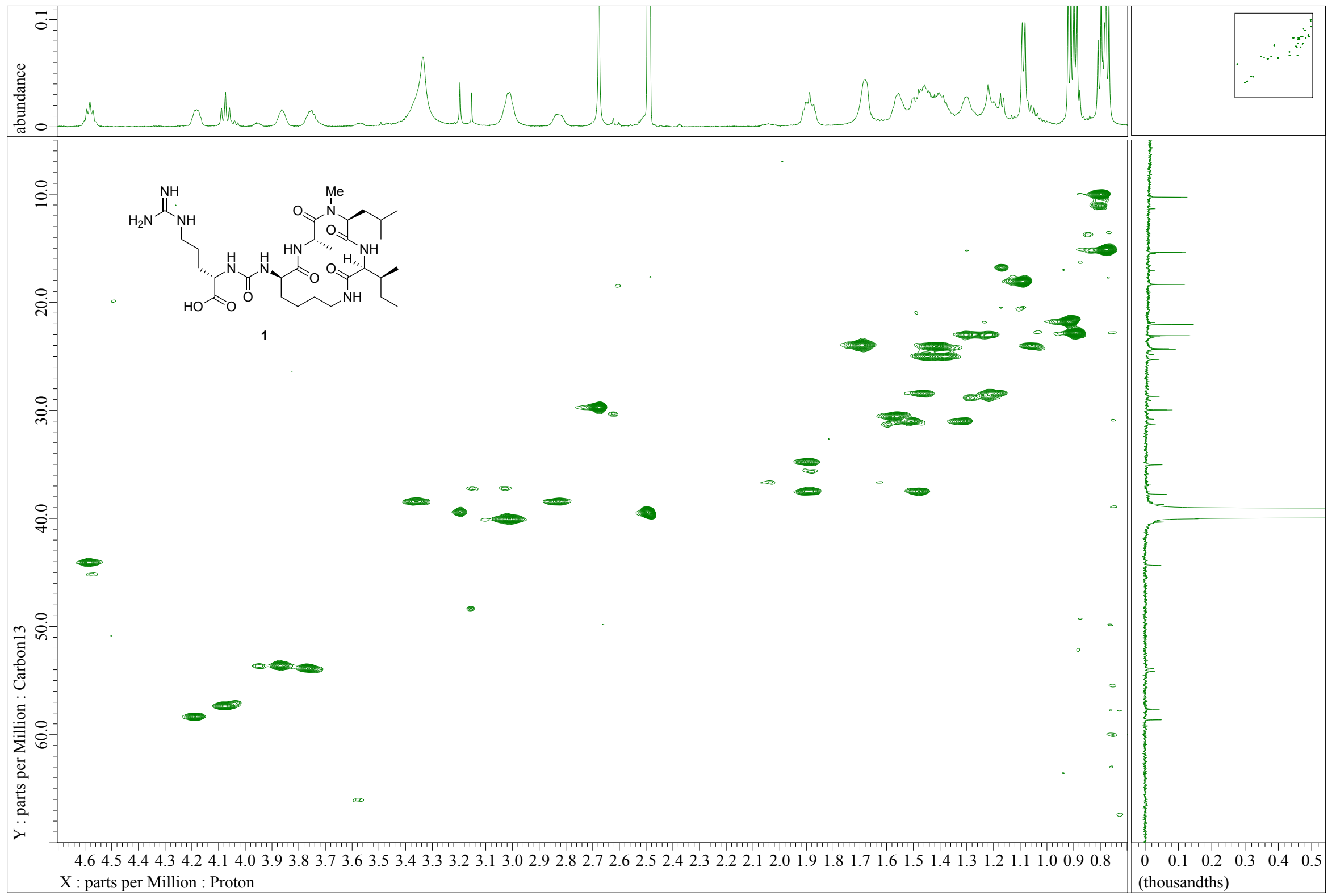

Figure S5. HSQC spectrum (600 MHz) of homophymamide A (1) in DMSO- $d_{6}$ 


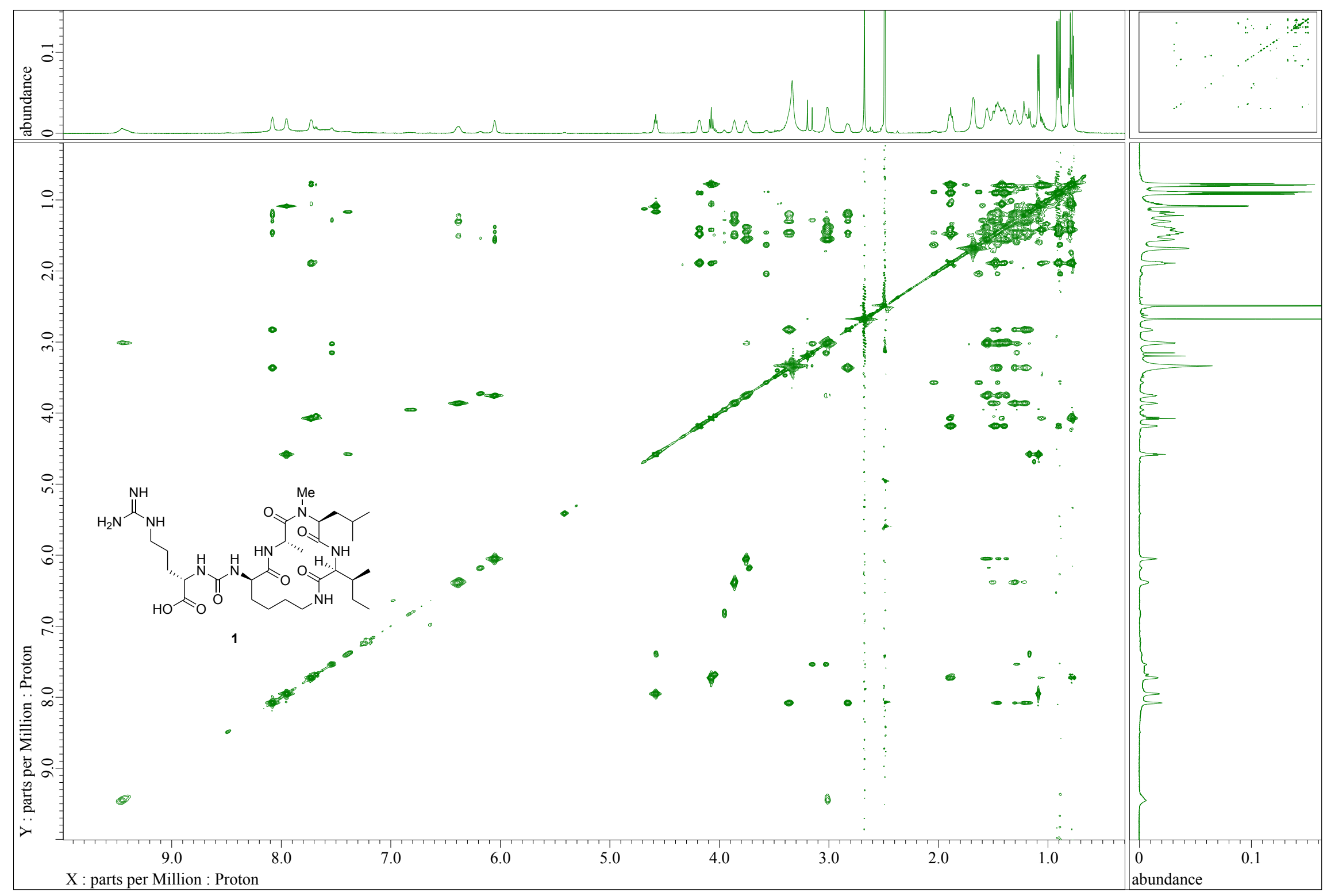

Figure S6. TOCSY spectrum (600 MHz) of homophymamide A (1) in DMSO- $d_{6}$ 


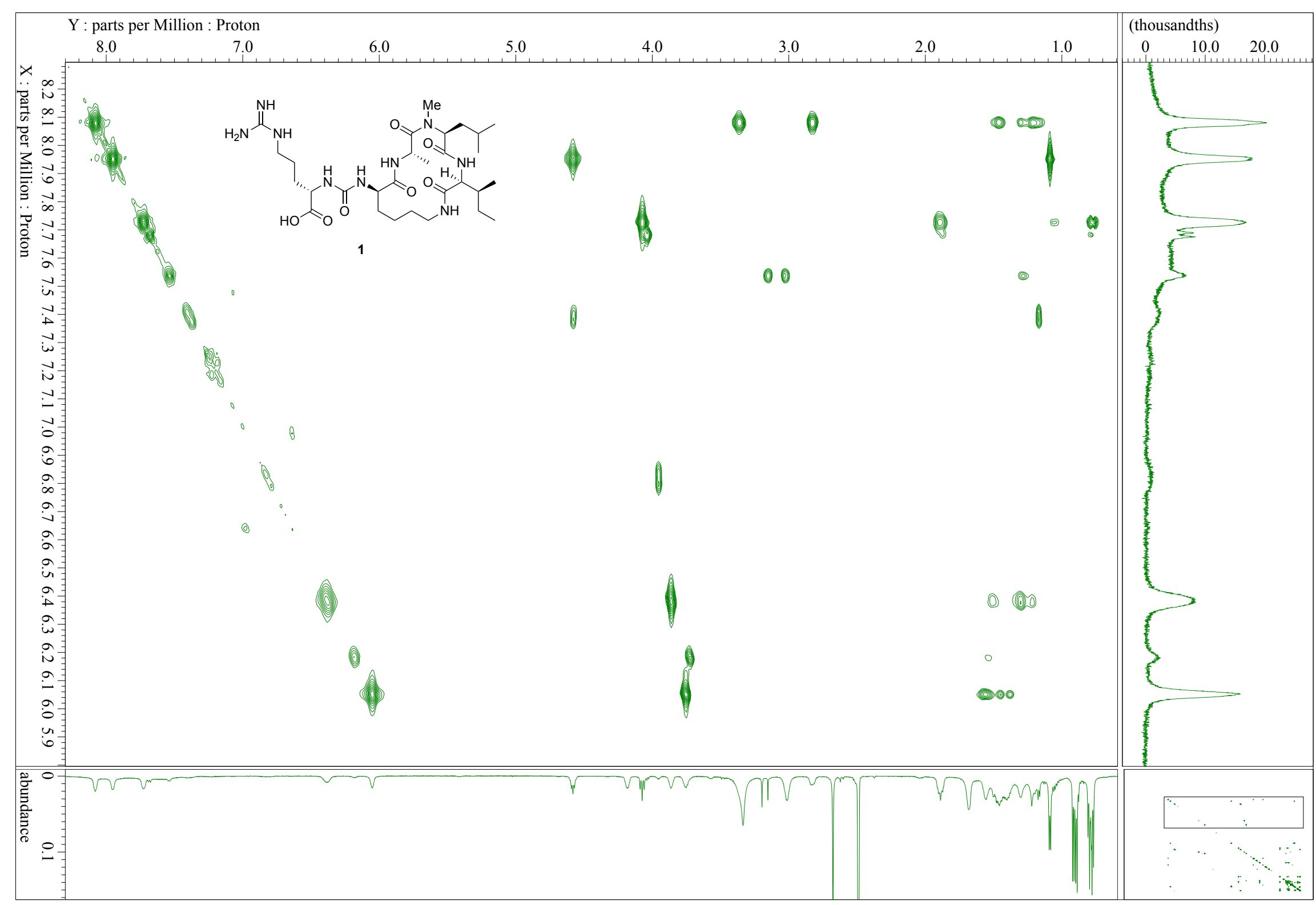

Figure S7. TOCSY spectrum (600 MHz) of homophymamide A (1) in DMSO- $d_{6}$ (amide proton region) 


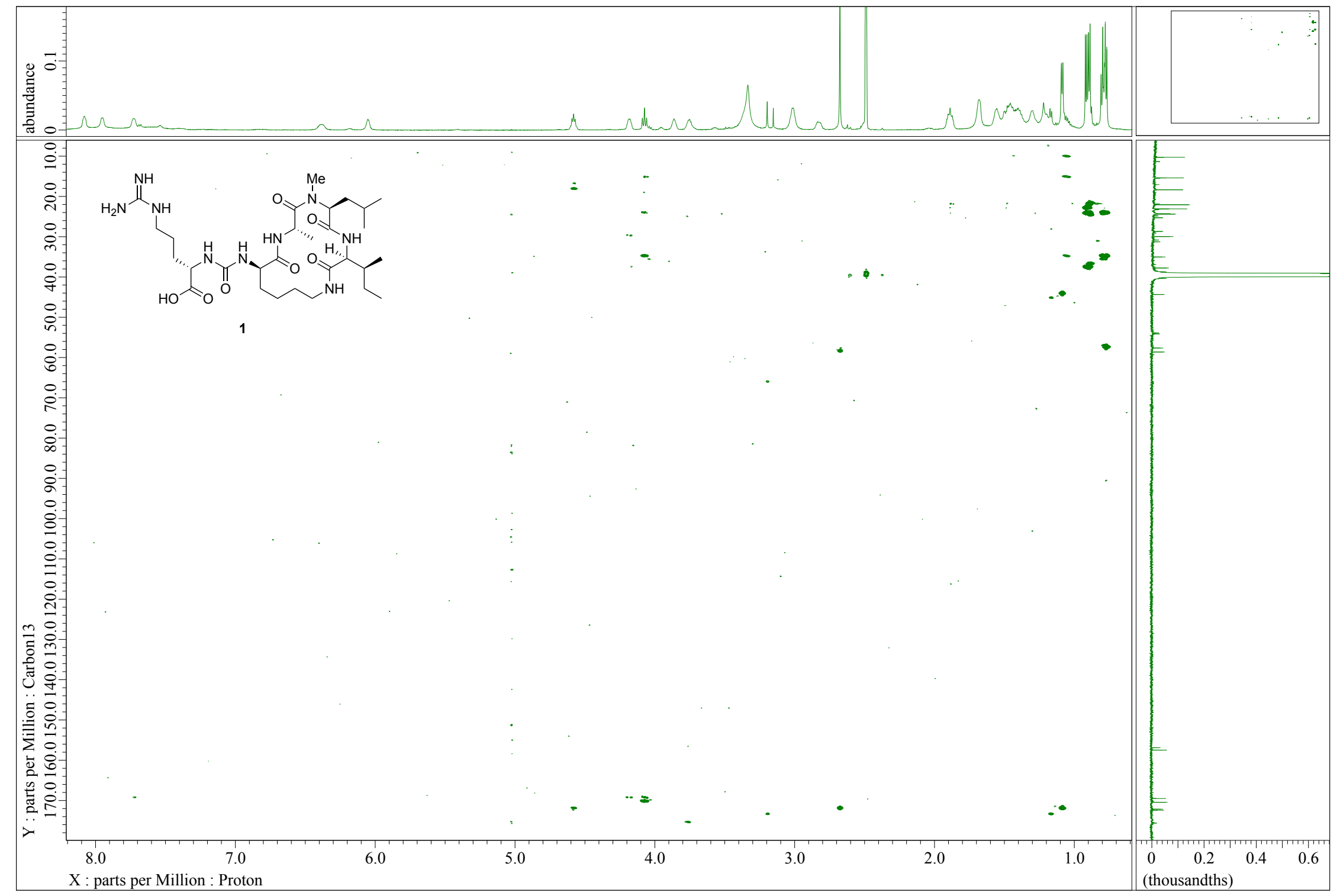

Figure S8. HMBC spectrum (600 MHz) of homophymamide A (1) in DMSO- $d_{6}$ 


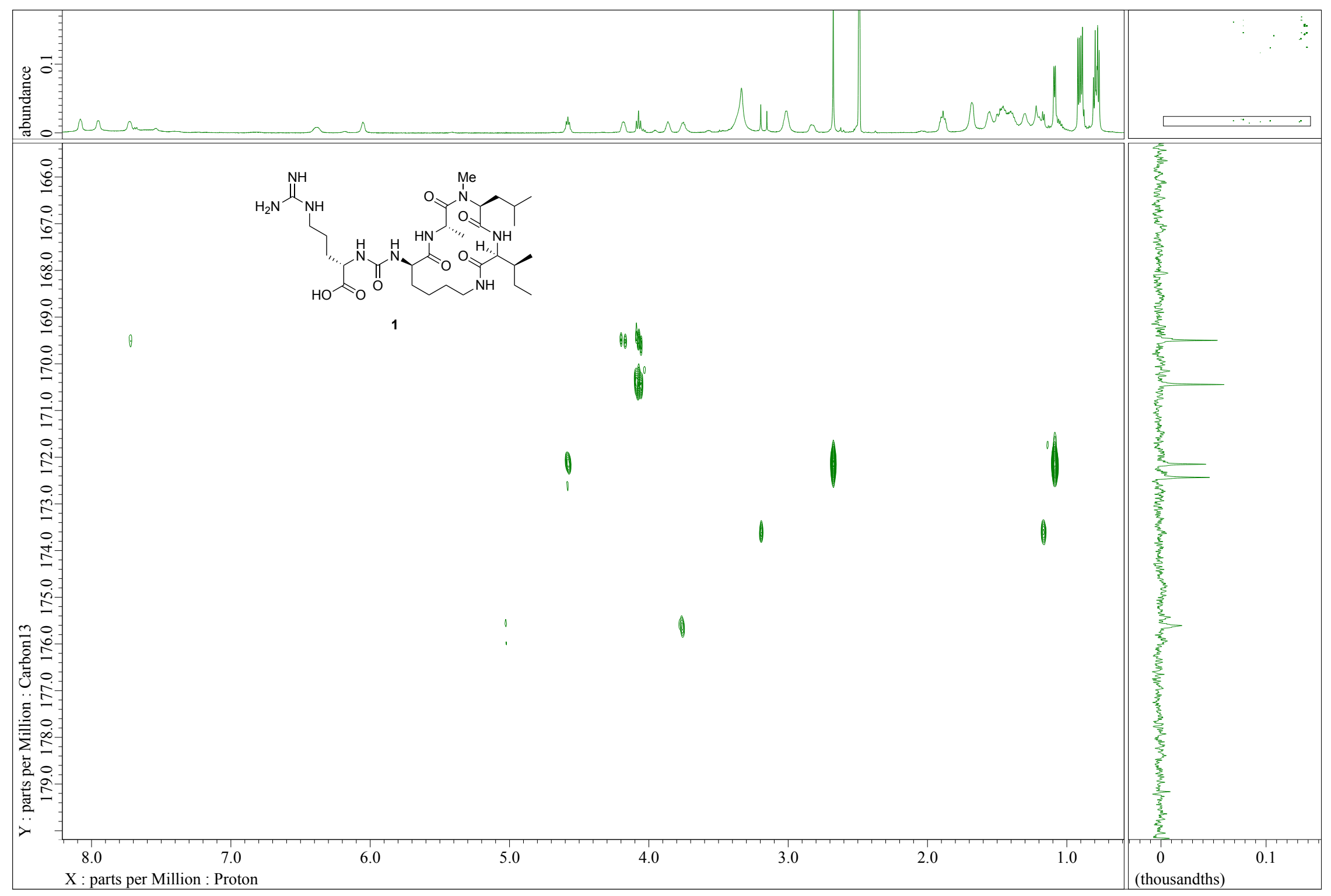

Figure S9. HMBC spectrum (600 MHz) of homophymamide A (1) in DMSO- $d_{6}$ (amide carbonyl region) 


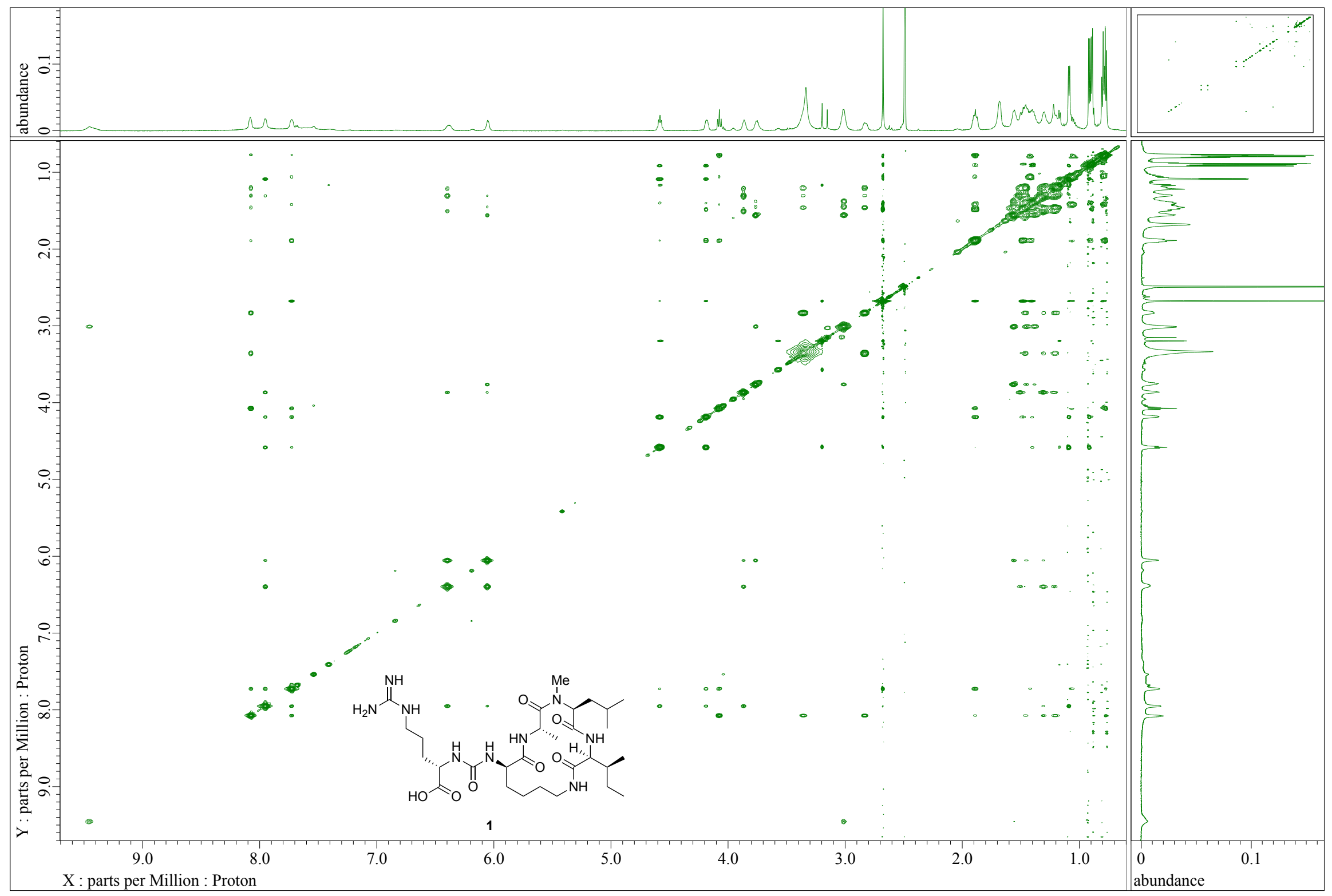

Figure S10. NOESY spectrum (600 MHz) of homophymamide A (1) in DMSO- $d_{6}$ 


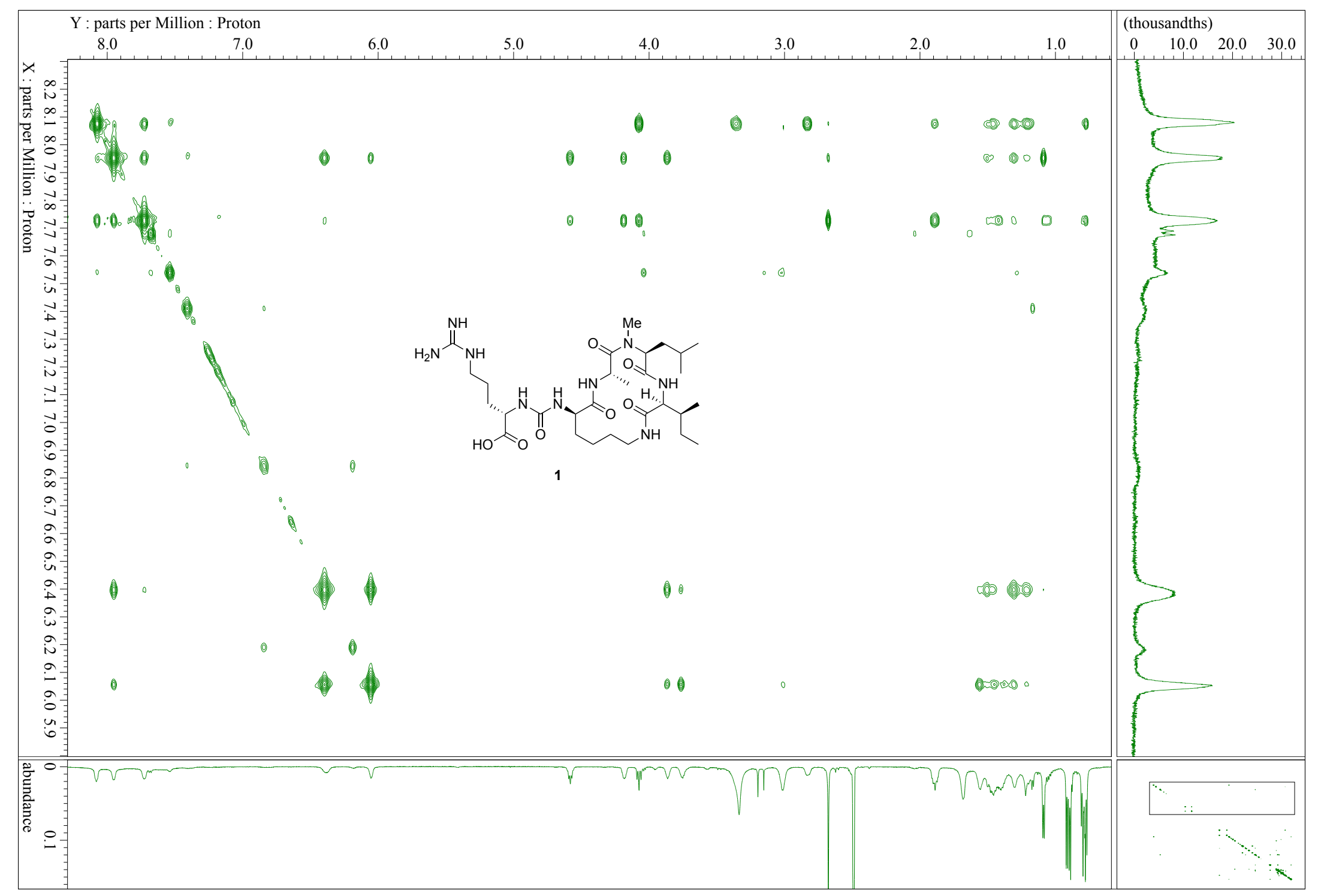

Figure S11. NOESY spectrum (600 MHz) of homophymamide A (1) in DMSO- $d_{6}$ (amide proton region) 


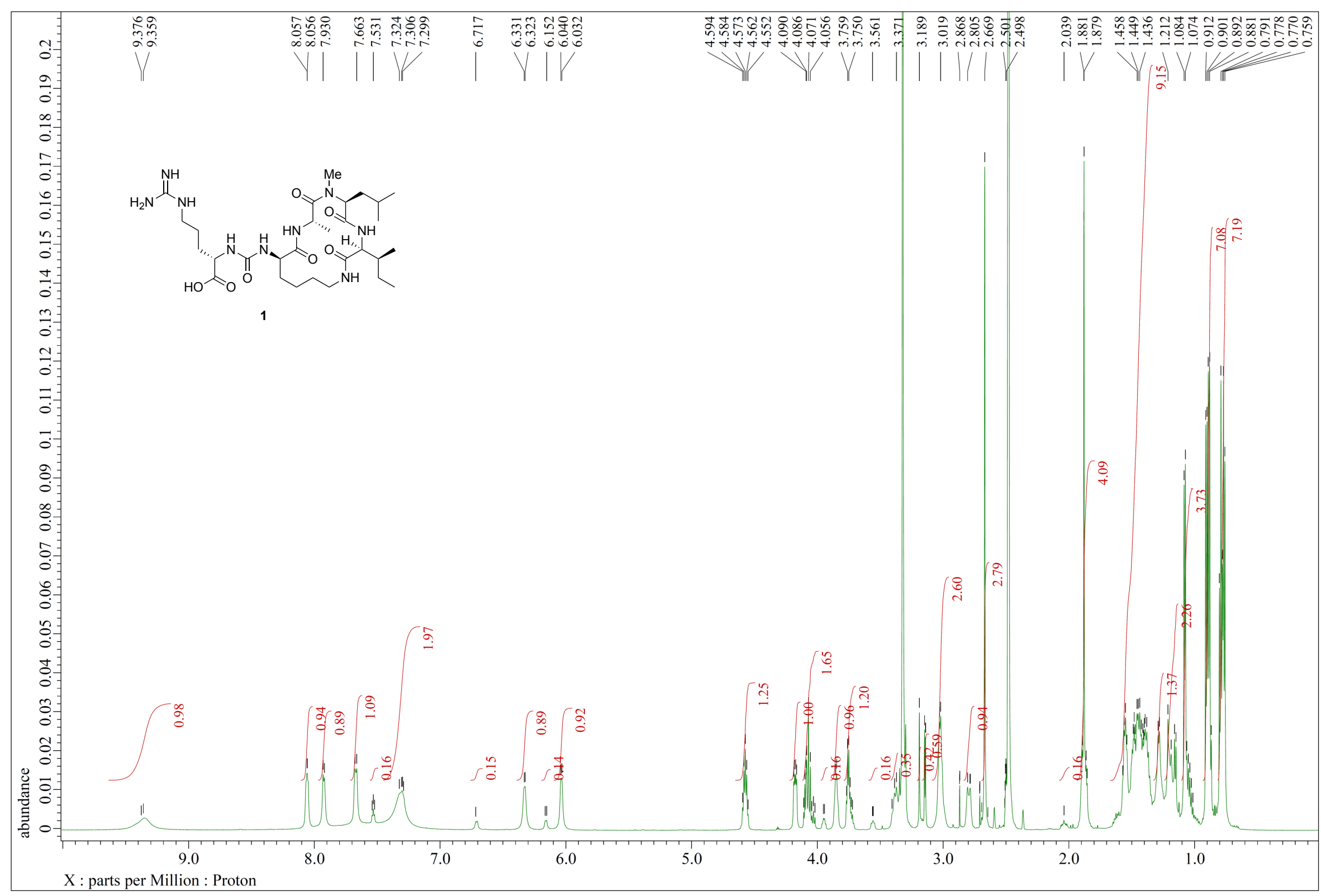

Figure S12. ${ }^{1} \mathrm{H}$ NMR spectrum (600 MHz) of synthetic homophymamide A (1) in DMSO- $d_{6}$ 


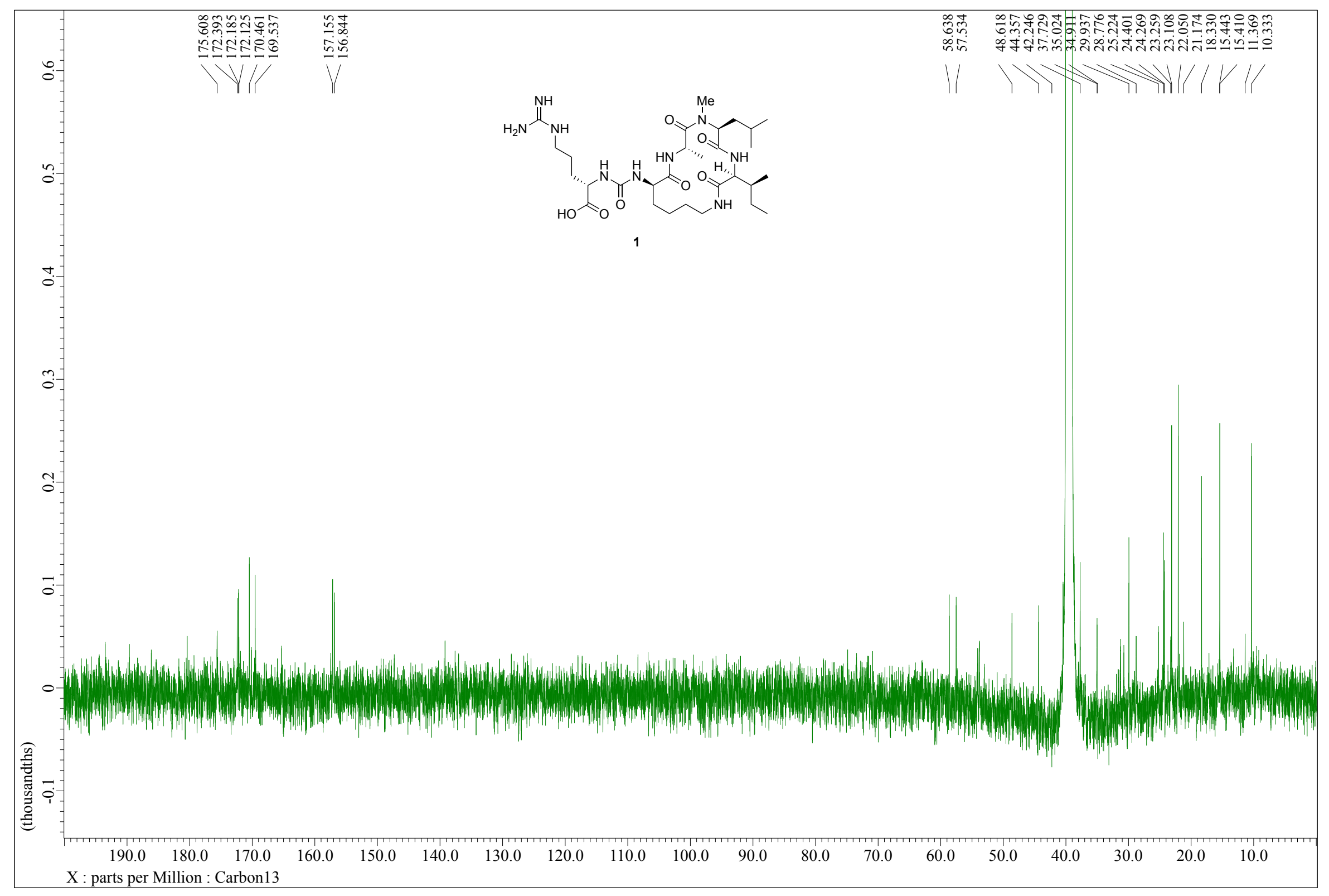

Figure S13. ${ }^{13} \mathrm{C}$ NMR spectrum $(150 \mathrm{MHz})$ of synthetic homophymamide A (1) in DMSO- $d_{6}$ 


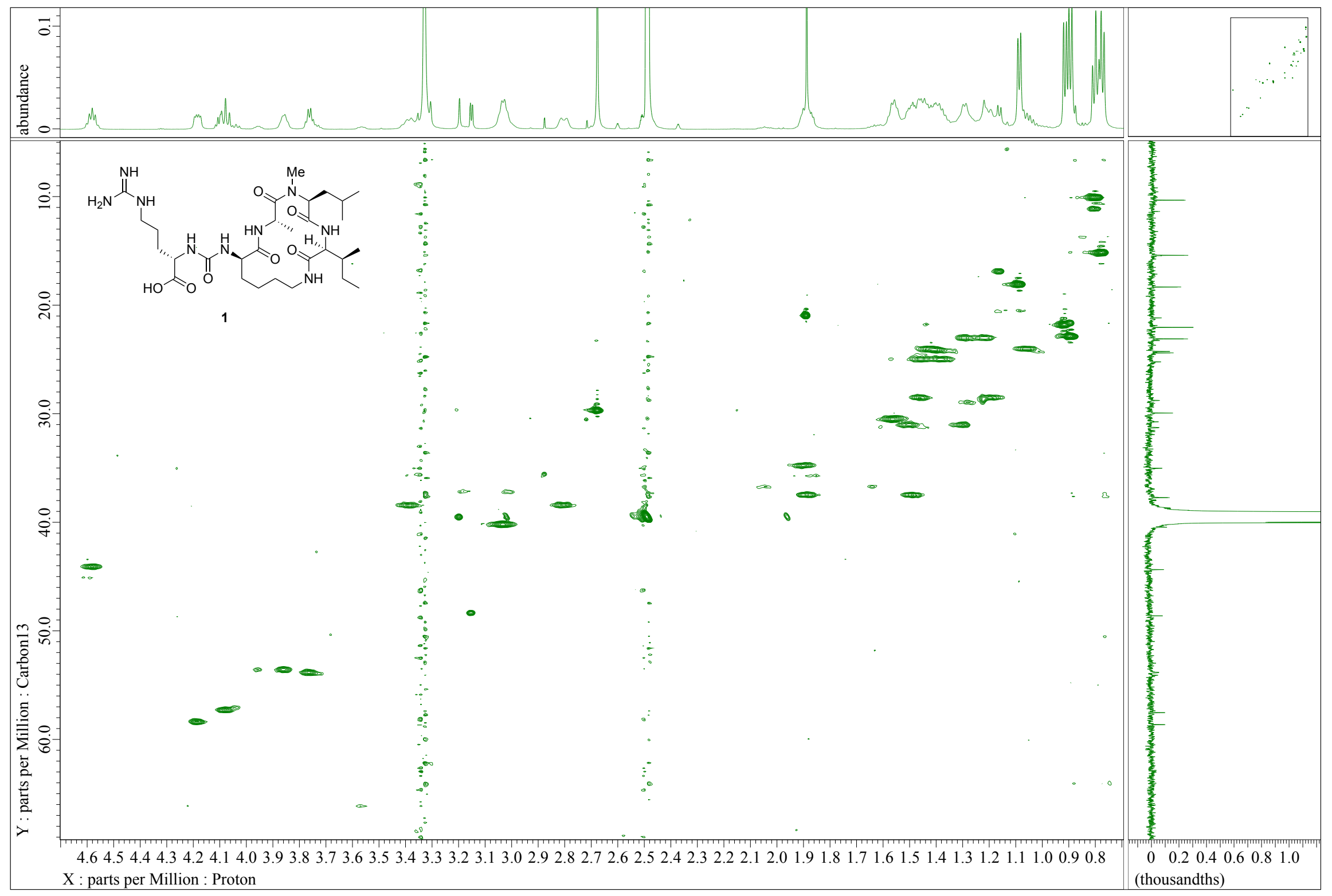

Figure S14. HSQC spectrum (600 MHz) of synthetic homophymamide A (1) in DMSO- $d_{6}$ 


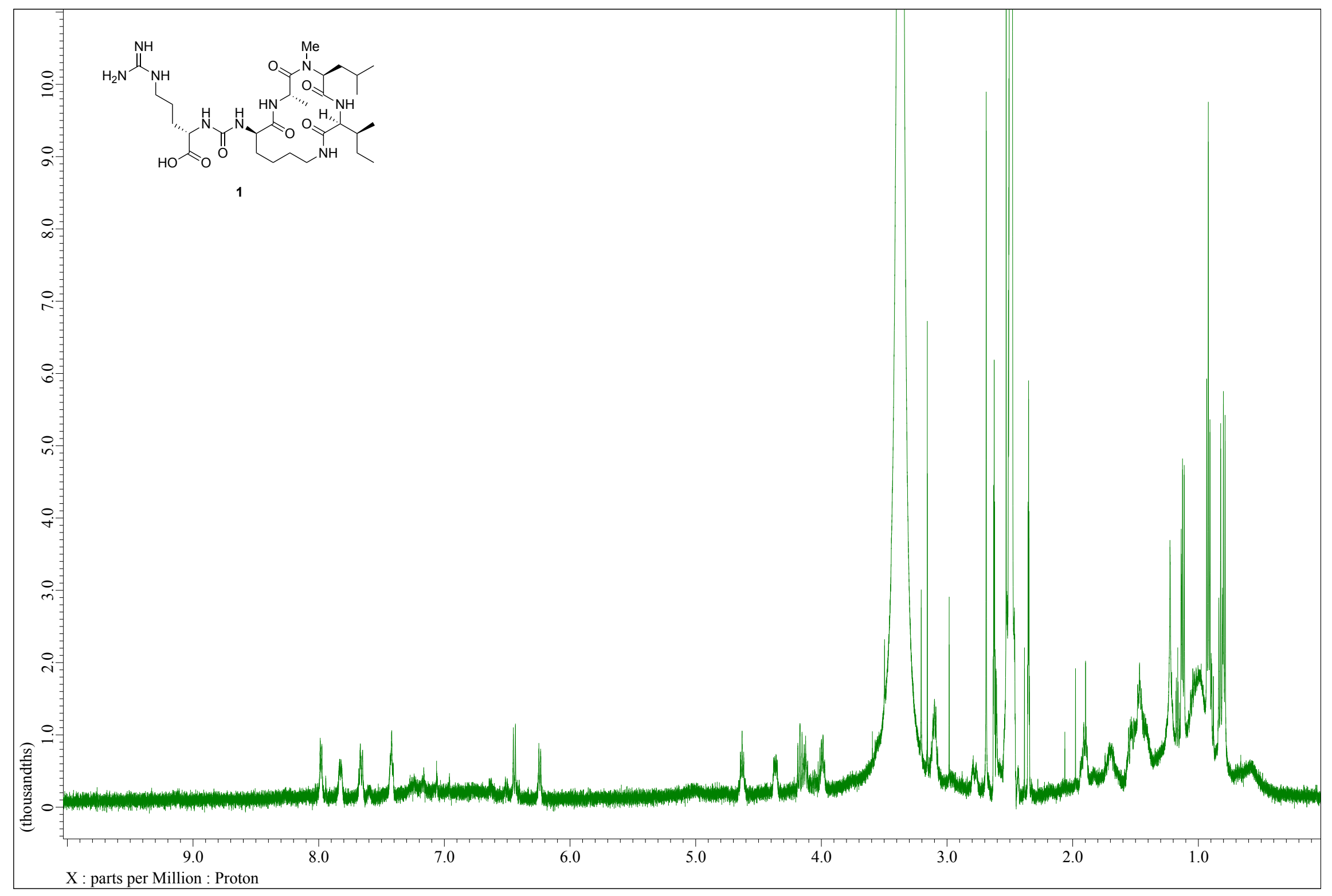

Figure S15. ${ }^{1} \mathrm{H}$ NMR spectrum (500 MHz) of a 1:1 mixture of natural product and synthetic homophymamide A (1) in DMSO- $d_{6}$ 


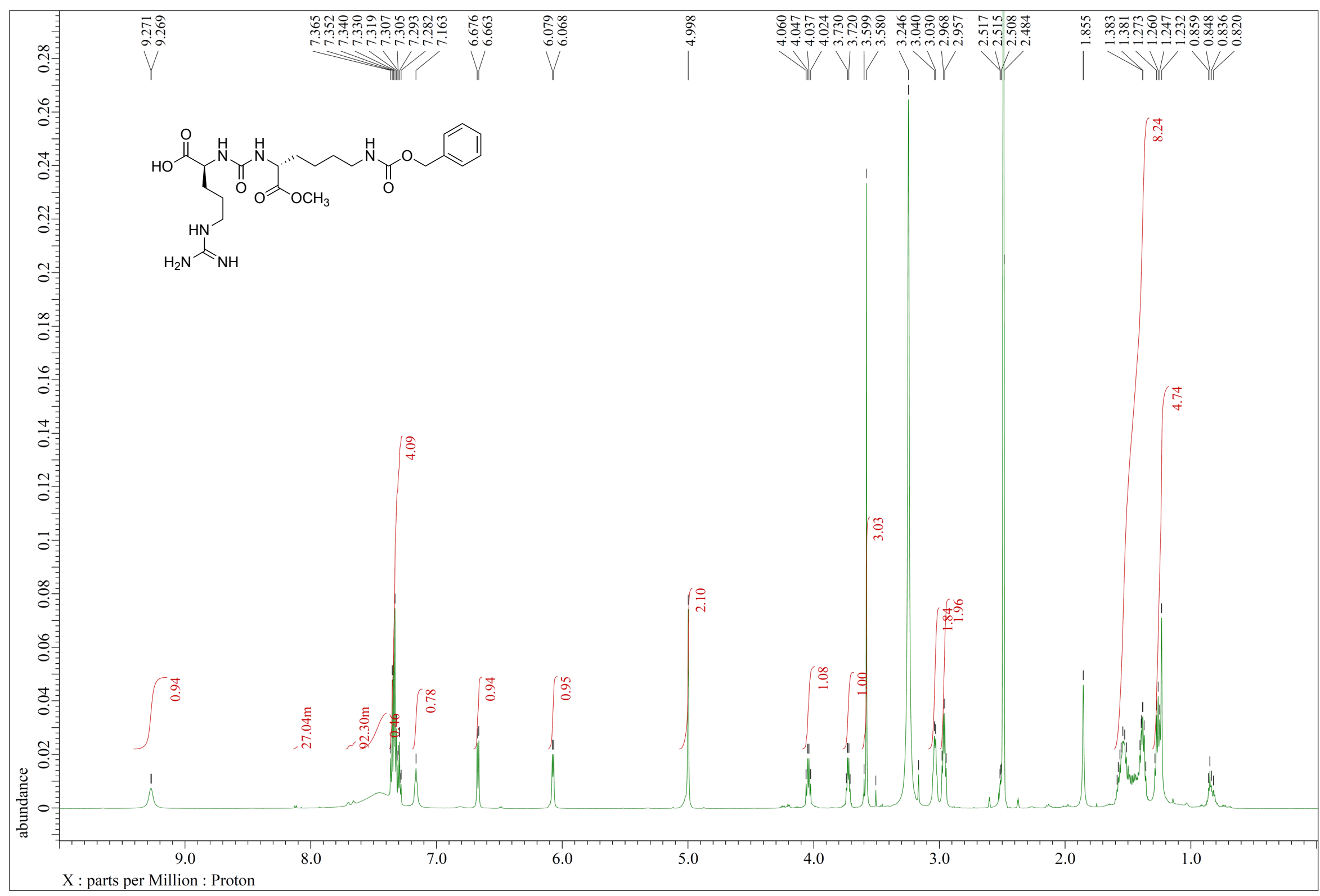

Figure S16. ${ }^{1} \mathrm{H}$ NMR spectrum $(600 \mathrm{MHz})$ of L-Arg(N $)$-CO-D-Lys(Z) methyl ester (3) in DMSO- $d_{6}$ 


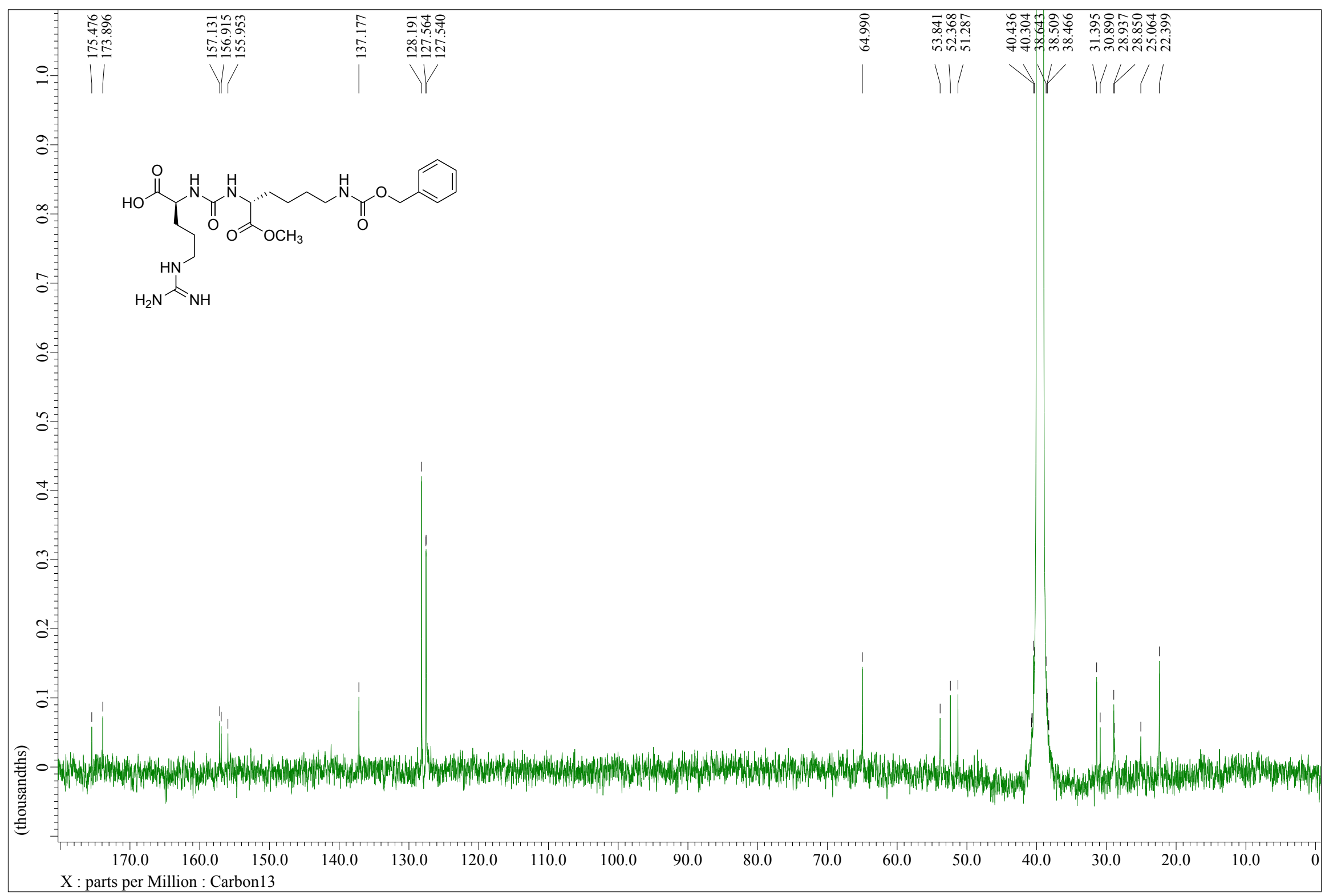

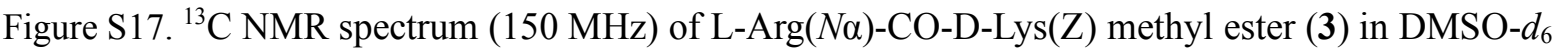




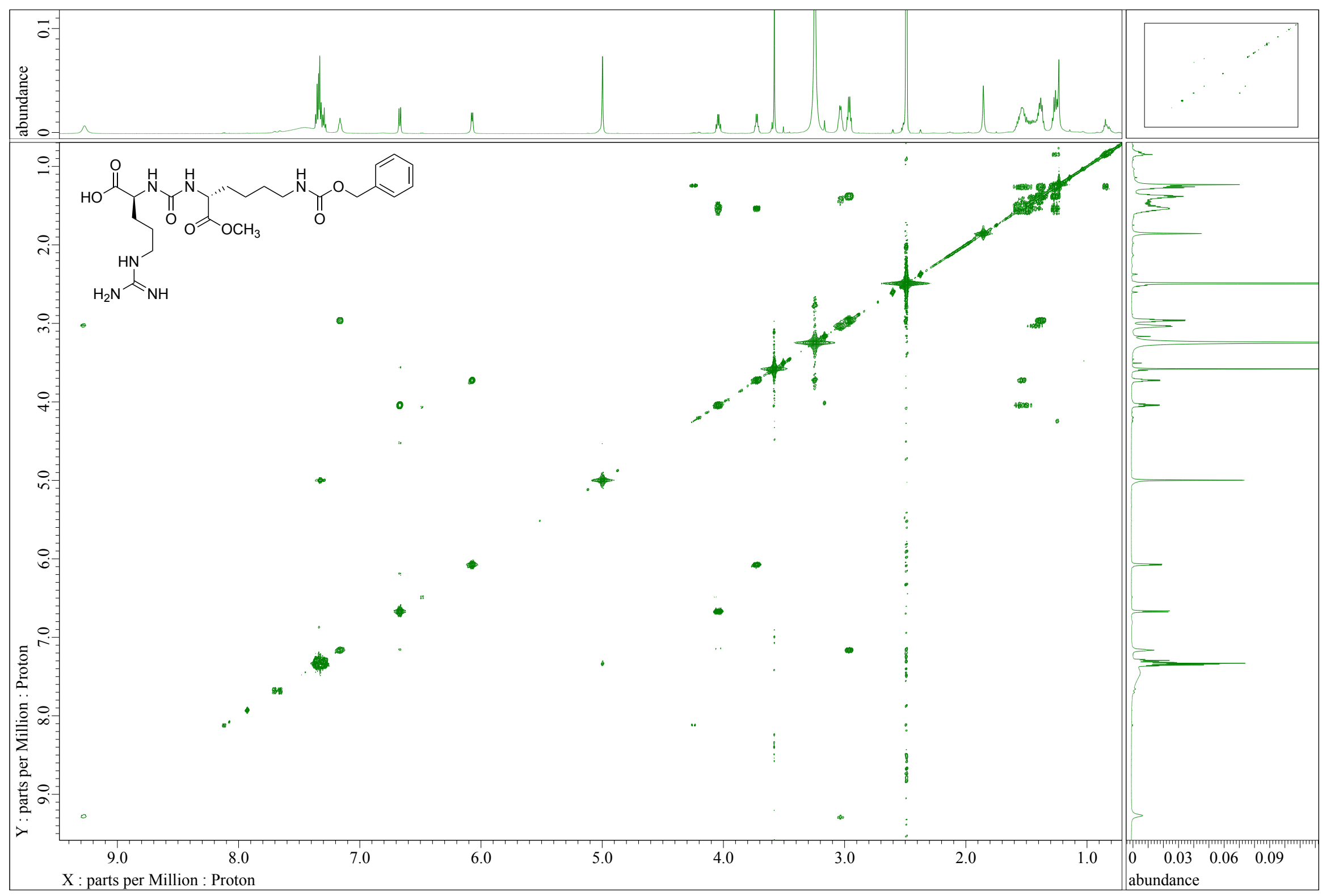

Figure S18. COSY spectrum (600 MHz) of L-Arg(Na)-CO-D-Lys(Z) methyl ester (3) in DMSO- $d_{6}$ 


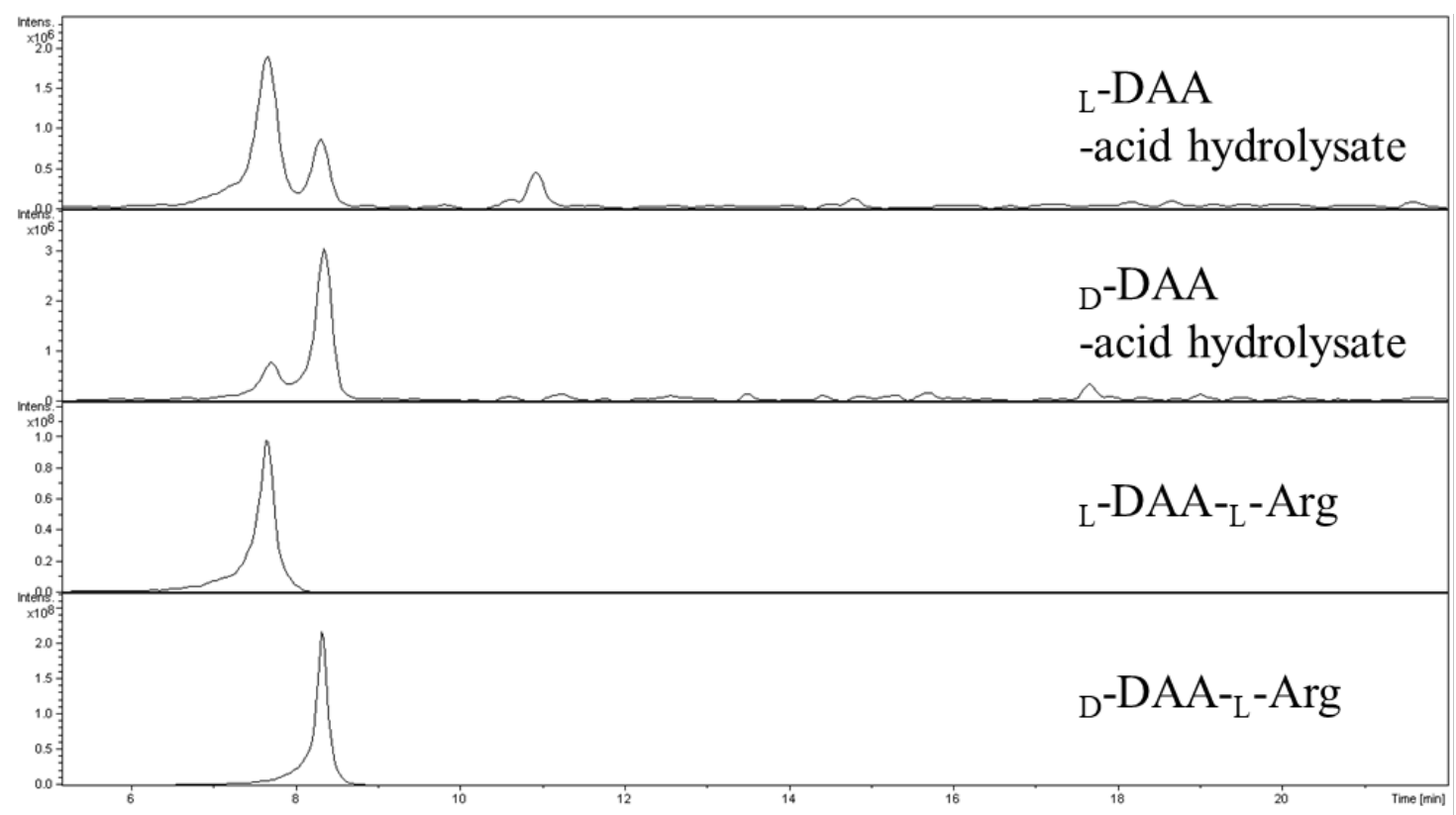

Figure S19. LCMS chromatogram of Marfey's derivatives

Chromatograms were monitored with ESIMS at $m / z 427.2[\mathrm{M}+\mathrm{H}]^{+}$.

L-DAA: 2-4-dinitrophenyl-5-L-alanine amide

D-DAA: 2-4-dinitrophenyl-5-D-alanine amide

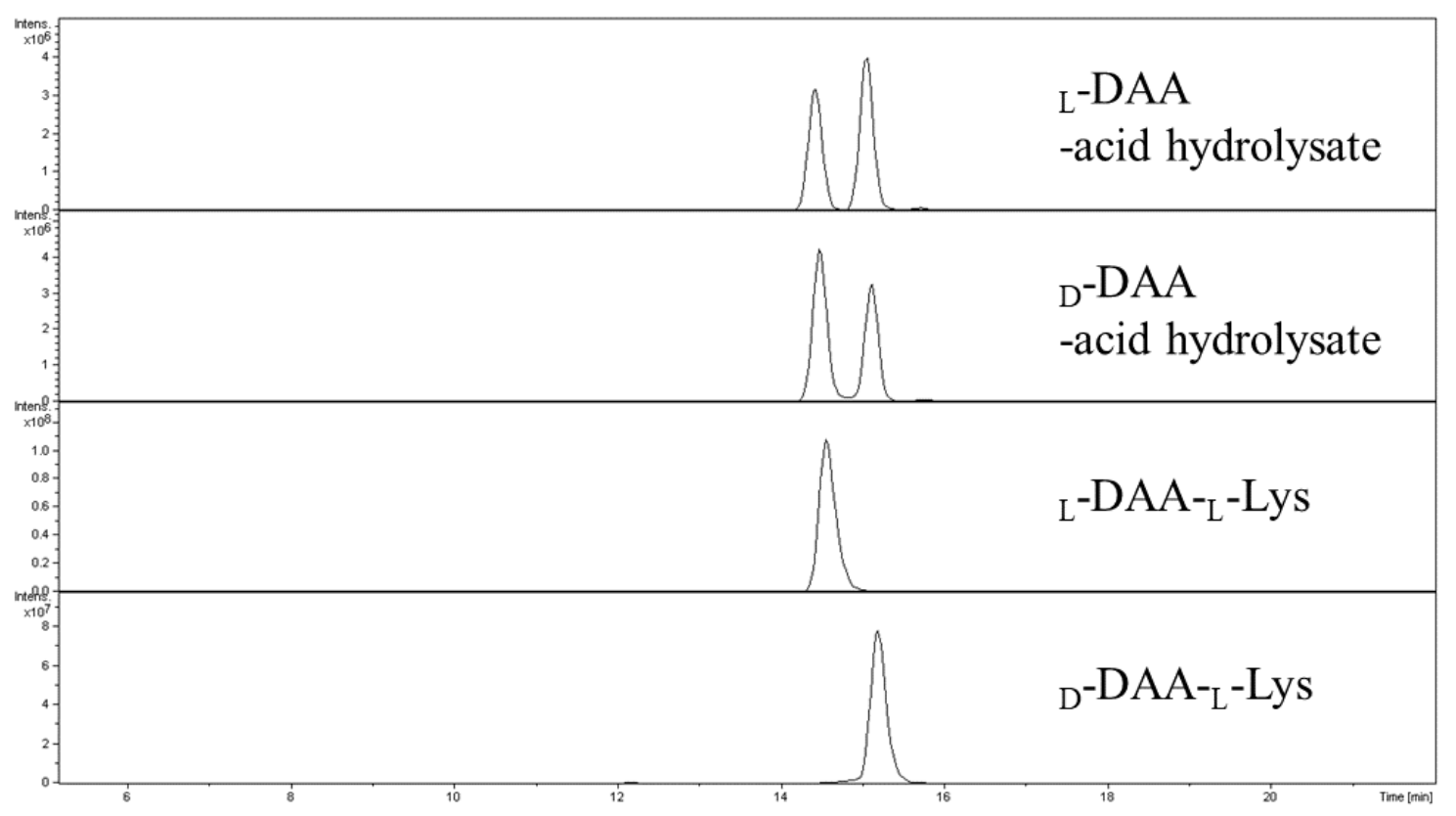

Figure S20. LCMS chromatogram of Marfey's derivatives

Chromatograms were monitored with ESIMS at $m / z 649.2[\mathrm{M}-\mathrm{H}]^{-}$. 


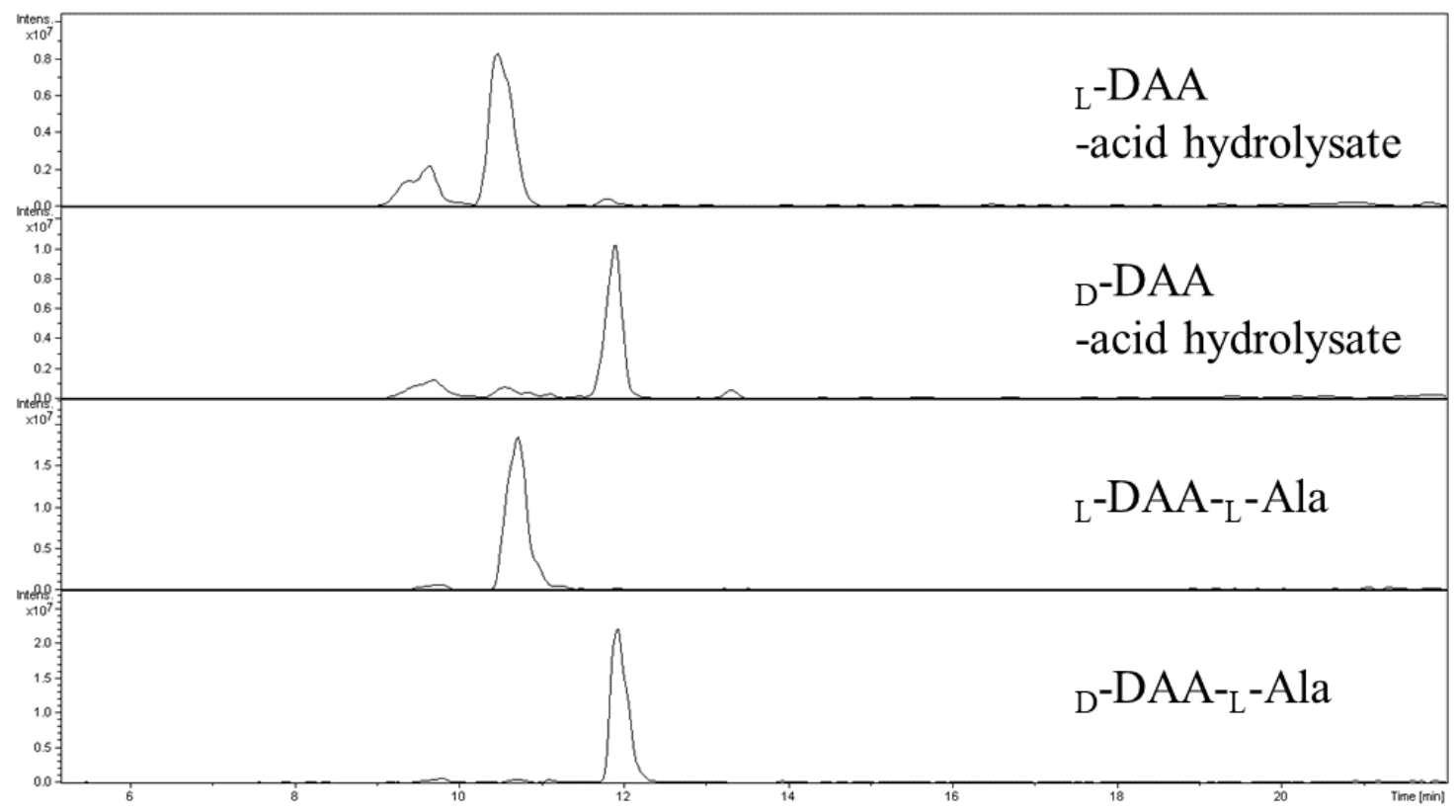

Figure S21. LCMS chromatogram of Marfey's derivatives

Chromatograms were monitored with ESIMS at $m / z 342.1[\mathrm{M}+\mathrm{H}]^{+}$.

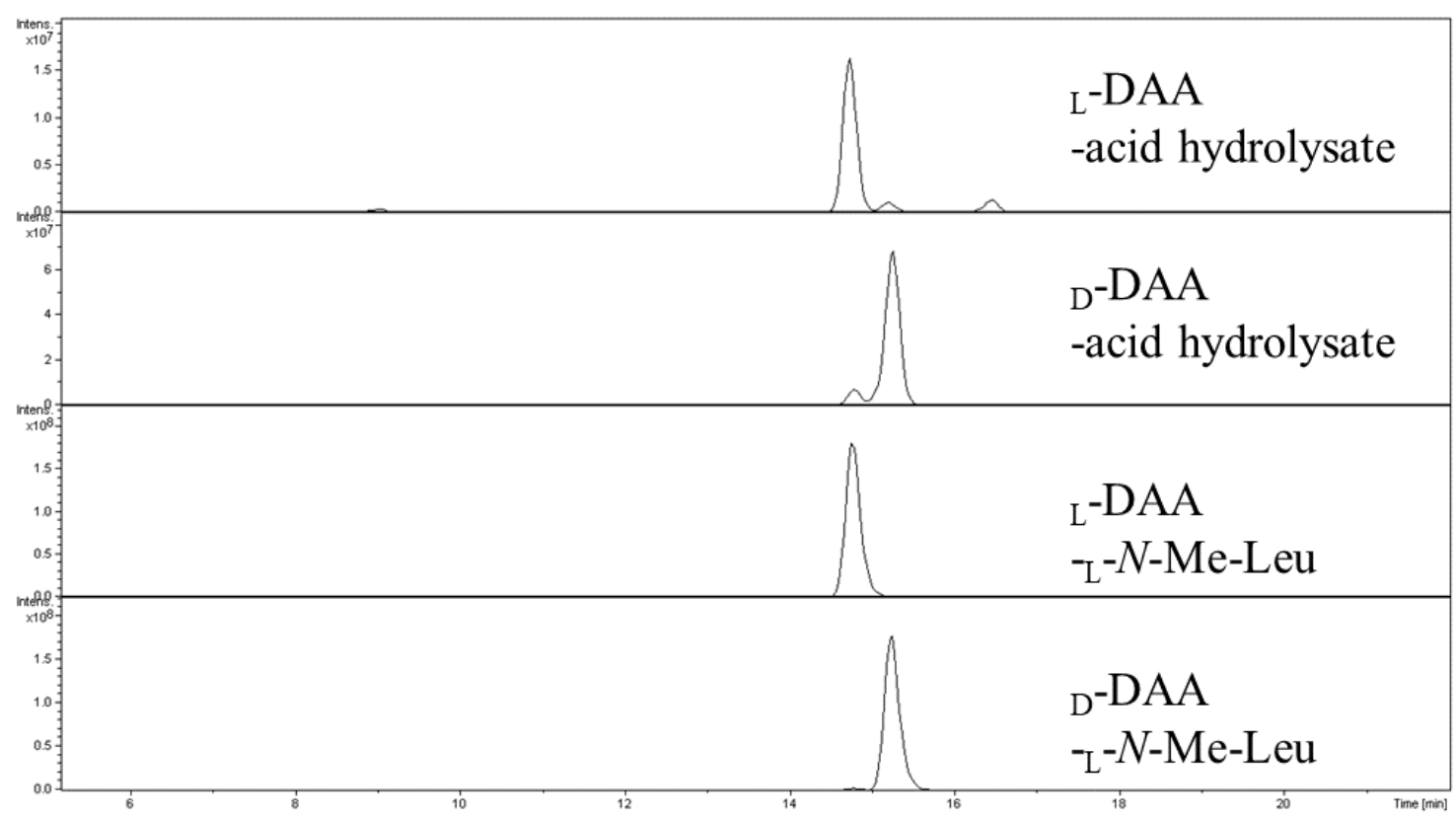

Figure S22. LCMS chromatogram of Marfey's derivatives

Chromatograms were monitored with ESIMS at $m / z 398.2[\mathrm{M}+\mathrm{H}]^{+}$. 


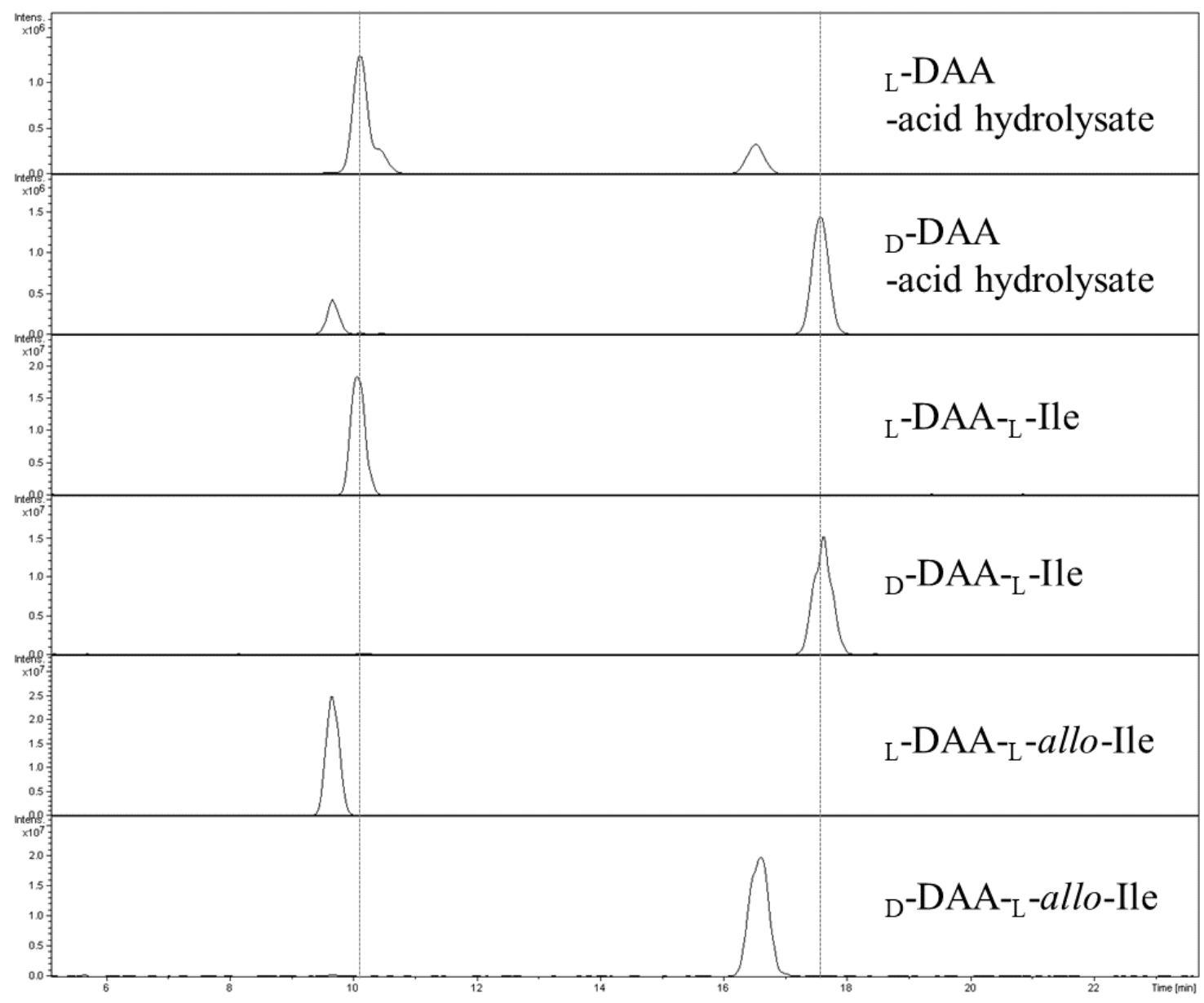

Figure S23 LCMS chromatogram of Marfey's derivatives Chromatograms were monitored with ESIMS at $m / z 382.2[\mathrm{M}-\mathrm{H}]^{-}$. 


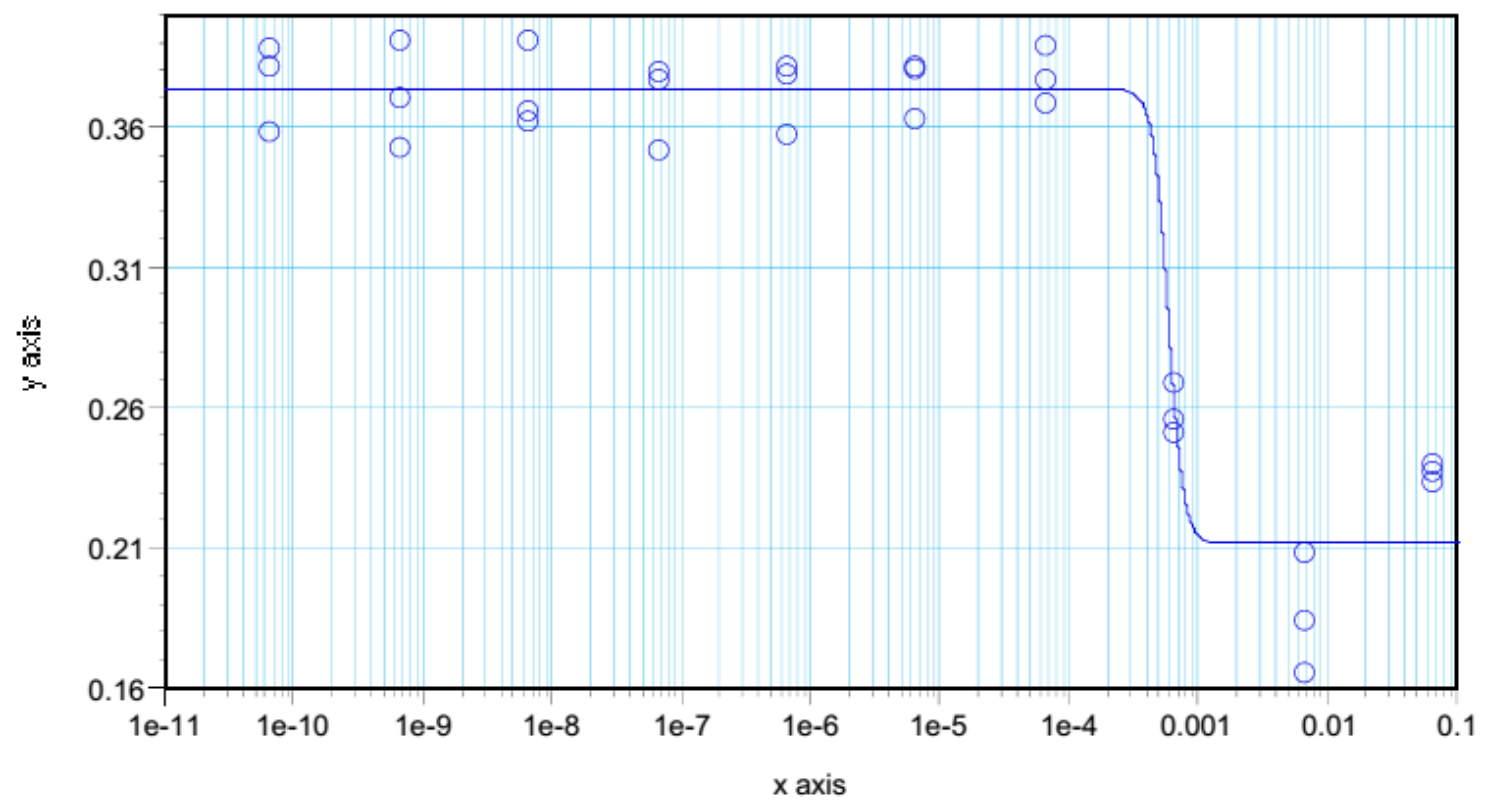

$\begin{array}{cccccc}y=\left((\mathrm{A}-\mathrm{D}) /\left(1+(\mathrm{x} / \mathrm{C})^{\wedge} \mathrm{B}\right)\right)+\mathrm{D}: & \underline{\mathrm{A}} & \underline{\mathrm{B}} & \underline{\mathrm{C}} & \underline{\mathrm{D}} & \underline{\mathrm{R}^{\wedge} 2} \\ \mathrm{O} \text { Plot\#1 (Group\#1: Dilution vs Values) } & 0.373 & 7.4 & 5.85 \mathrm{e}-4 & 0.211 & 0.945\end{array}$

Figure S24. Carboxypeptidase B inhibition assay of 1 
<smiles>COC(=O)[C@H](N)CCCCNC(=O)OCc1ccccc1</smiles><smiles>N=C(N)NCCCC(N=C=O)C(=O)Oc1ccccc1</smiles><smiles>COC(=O)C(CCCCNC(=O)OCc1ccccc1)NC(=O)NC(CCCNC(=N)N)C(=O)Oc1ccccc1</smiles><smiles>COC(=O)[C@H](CCCCNC(=O)OCc1ccccc1)NC(=O)NC(CCCNC(=N)N)C(=O)O</smiles><smiles>N=C(N)NCCCC(C(=O)O)N1C(=O)N[C@H](CCCCN)C1=O</smiles>

L-Arg(N $\alpha)$-CO-D-Lys(Z) methyl ester (3)

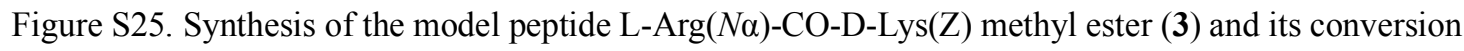
to the hydantoin 4 . 


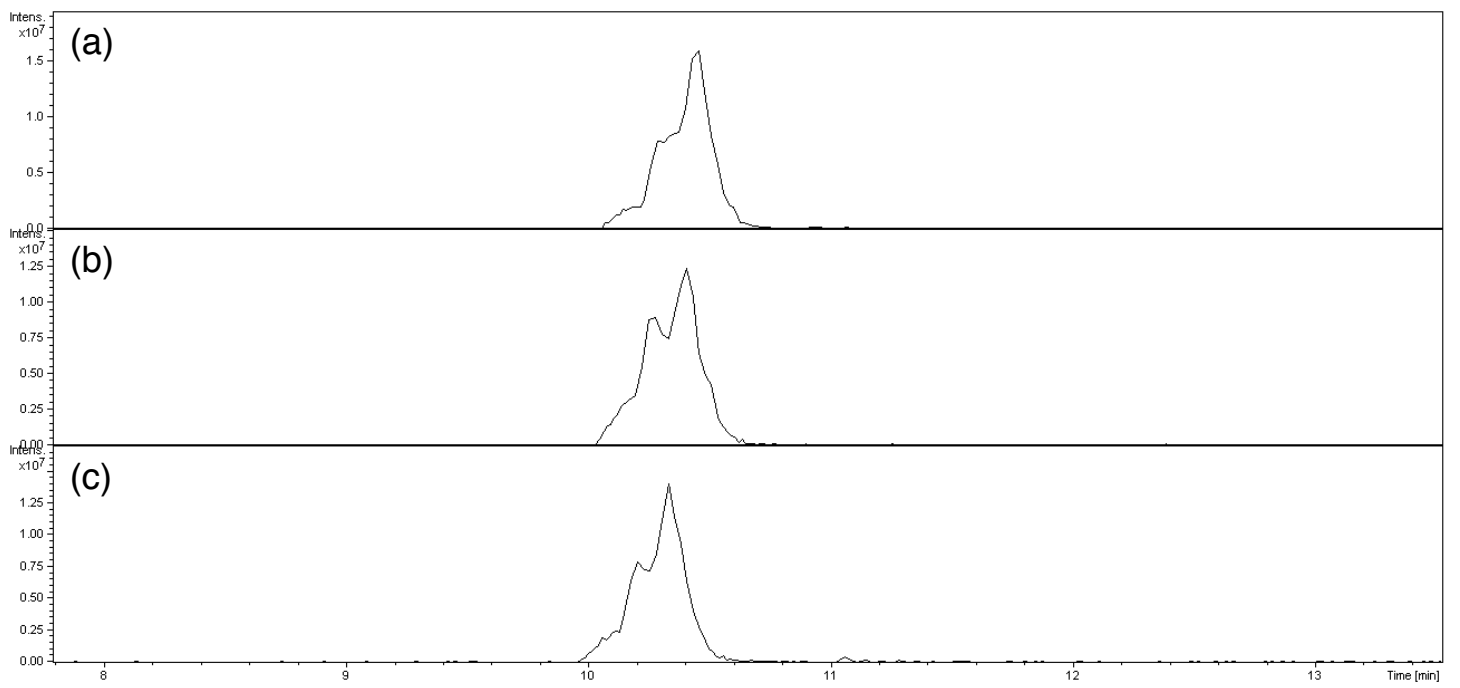

Figure S26. LCMS chromatogram of the Marfey's derivative of hydantoin 4. Chromatograms were monitored with ESIMS at $m / z 581.2[\mathrm{M}+\mathrm{H}]^{+}$. (a) Acid hydrolysate of 1. (b) Acid hydrolysate of 3 . (c) Co-injection. 